\title{
Determining novel candidate anti-hepatocellular carcinoma drugs using interaction networks and molecular docking between drug targets and natural compounds of SiNiSan
}

\author{
Qin Zhang Equal first author, 1 , Zhangying Feng ${ }^{\text {Equal first author, } 2, \text { Mengxi Gao }^{1} \text {, Liru Guo }}{ }^{\text {Corresp. } 1}$ \\ ${ }^{1}$ The Fourth Hospital of Hebei Medical University, Department of General Medicine, Shijiazhuang, Hebei, China \\ 2 The Fourth Hospital of Hebei Medical University, Department of Clinical Pharmacology, Shijiazhuang, Hebei, China \\ Corresponding Author: Liru Guo \\ Email address: qkglrdoctor@126.com
}

Background: SiNiSan (SNS) is an ancient traditional Chinese medicine (TCM) used to treat liver and spleen deficiencies. We studied the unique advantages of using SNS to treat hepatocellular carcinoma ( $\mathrm{HCC}$ ) with multiple components and targets to determine its potential mechanism of action. Methods: The active compounds from the individual herbs in the SNS formula and their targets were mined from Traditional Chinese Medicine Systems Pharmacology Database (TCMSP). HCC-associated targets were collected from the TCGA and GEO databases and samples were collected from patients with stage III hepatocellular carcinoma. A compound-disease target network was constructed, visualized, and analyzed using Cytoscape software. We built a protein-protein interaction (PPI) network using the String database. We enriched and analyzed key targets using GSEA, GO, and KEGG in order to explore their functions. Autodock software was used to simulate the process of SNS molecules acting on HCC targets. Results: 113 candidate compounds were taken from SNS, and 64 of the same targets were chosen from HCC and SNS. The predominant targets genes were PTGS2, ESR1, CHEK1, CCNA2, NOS2 and AR; kaempferol and quercetin from SNS were the principal ingredients in HCC treatment. The compounds may work against HCC due to a cellular response to steroid hormones and histone phosphorylation. The P53 signaling pathway was significantly enriched in the gene set GSEA enrichment analysis and differential gene KEGG enrichment analysis.

Conclusions: Our results showed that the SNS component has a large number of stage III HCC targets. Among the targets, the sex hormone receptors, the AR and ESR1 genes, are the core targets of SNS component and the most active proteins in the PPI network. In addition, quercetin, which has the most targets, can act on the main targets (BAX, CDK1, CCNB1, SERPINE1, CHEK2, and IGFBP3) of the P53 pathway to treat HCC. 
1 Determining novel candidate anti-hepatocellular carcinoma drugs using interaction

2 networks and molecular docking between drug targets and natural compounds of SiNiSan

3

4

5 Qin Zhang ${ }^{1, \Delta}$, Zhangying Feng ${ }^{2, \Delta}$, Mengxi Gao ${ }^{1}$, Liru Guo ${ }^{1 *}$

6

7

$8{ }^{1}$ The Fourth Hospital of Hebei Medical University, Department of General Medicine,

9 Shijiazhuang, Hebei, China

$10{ }^{2}$ The Fourth Hospital of Hebei Medical University, Department of Clinical Pharmacology,

11 Shijiazhuang, Hebei, China

${ }^{\Delta}$ These authors contribute equally in this work.

Corresponding Author:

18 Liru GUO

1912 Health Road, Shijiazhuang, Hebei, 050011, China

20 Email address: qkglrdoctor@126.com

21

22 


\section{Abstract}

30 Background: SiNiSan (SNS) is an ancient traditional Chinese medicine (TCM) used to treat

31 liver and spleen deficiencies. We studied the unique advantages of using SNS to treat

32 hepatocellular carcinoma (HCC) with multiple components and targets to determine its potential

33 mechanism of action.

34 Methods: The active compounds from the individual herbs in the SNS formula and their targets

35 were mined from Traditional Chinese Medicine Systems Pharmacology Database (TCMSP).

36 HCC-associated targets were collected from the TCGA and GEO databases and samples were

37 collected from patients with stage III hepatocellular carcinoma. A compound-disease target

38 network was constructed, visualized, and analyzed using Cytoscape software. We built a protein-

39 protein interaction (PPI) network using the String database. We enriched and analyzed key

40 targets using GSEA, GO, and KEGG in order to explore their functions. Autodock software was

41 used to simulate the process of SNS molecules acting on HCC targets.

42 Results: 113 candidate compounds were taken from SNS, and 64 of the same targets were

43 chosen from HCC and SNS. The predominant targets genes were PTGS2, ESR1, CHEK1,

44 CCNA2, NOS2 and AR; kaempferol and quercetin from SNS were the principal ingredients in

45 HCC treatment. The compounds may work against HCC due to a cellular response to steroid

46 hormones and histone phosphorylation. The P53 signaling pathway was significantly enriched in

47 the gene set GSEA enrichment analysis and differential gene KEGG enrichment analysis.

48 Conclusions: Our results showed that the SNS component has a large number of stage III HCC

49 targets. Among the targets, the sex hormone receptors, the AR and ESR1 genes, are the core

50 targets of SNS component and the most active proteins in the PPI network. In addition,

51 quercetin, which has the most targets, can act on the main targets (BAX, CDK1, CCNB1,

52 SERPINE1, CHEK2, and IGFBP3) of the P53 pathway to treat HCC.

\section{Introduction}

55 Hepatocellular carcinoma (HCC) is an extremely malignant type of tumor that accounts for the 56 majority of primary liver cancer cases worldwide. The increased incidence rate of HCC makes it

57 the leading cause of death among patients with cirrhosis (Forner et al., 2018). Liver cancer is the 
58 sixth most common neoplasm and the fourth leading cause of cancer death according to the 59 GLOBOCAN 2018 report issued by the International Agency for Research (IARC) (Ferlay et al., 60 2019).

61 HCC develops in stages and is caused by a combination of factors. Determining the 62 molecular mechanisms of HCC is complicated due to the physiological functions of the liver 63 (Pan et al., 2011). The following mechanisms have been confirmed: activation of proto64 oncogenes, such as N-ras and HBVx (Cheng et al., 2014; Kudo, 2011); inactivation of the tumor 65 suppressor genes, such as p53, Rb, p21 and PTEN (Sandra \& Jean-Charles, 2020; Maheshkumar 66 et al., 2019); abnormal activation of multiple molecular signaling pathways; and the expression of HCC-associated proteins. HCC treatments focus on increasing survival while maintaining a high quality of life. The treatments include surgery, interventional therapy, chemotherapy, and 69 70 71 72 73 combination therapy. Surgical treatments include hepatectomy, liver transplantation, and local ablative therapies. However, these are only used for early-stage liver cancer and tumors that are localized and not metastasized (Villanueva, 2019). Vascular interventional therapy mainly consists of transarterial chemoembolization (TACE) and transcatheter arterial infusion (TAI), which can effectively control the growth of HCC cells. However, there is no standardized staging or guidelines for interventional therapy ischemia, and hypoxia in tumor tissues after TACE therapy may lead to the increase of hypoxia-inducible factors, resulting in the high expression of VEGF in residual tumors, and eventually causing cancer recurrence and metastasis.

Chemotherapy is an important option for HCC treatment but is limited by its toxic side effects. An effective systematic treatment is required for patients with terminal-stage liver cancer or patients with severe underlying disease to act on multiple mechanisms and control tumor progression (Grandhi et al., 2016). Tumor staging is a crucial step in the treatment of HCC. TNM (Tumour, Node, Metastasis) and BCLC (The Barcelona Clinic Liver Cancer) are currently the most widely used evaluation systems, combined with the HCC study published in the Lancet in 2018 and the 2016 version of the AASLD (American Association for the Study of Liver Diseases) guideline (Forner et al., 2018; Heimbach et al., 2018). Patients with early stage HCC that tumor diameters $\leqslant 5 \mathrm{~cm}(\mathrm{~T} 1, \mathrm{~T} 2$ or $\mathrm{BCLC} 0, \mathrm{BCLCA})$ can benefit from resection, transplantation, and ablation treatments, for advanced HCC, the selection of treatment type depends on the extent of invasion of macrovascular, metastasis, and the physical condition of the patient, systemic treatment and the best supportive care may be the best choice, and this is 
89

90

91

92

93

94

95

96

97

98

99

100

101

102

103

104

105

106

107

108

109

110

111

112

113

114

115

116

117

118

119

exactly what we hope TCM achieves. Therefore, this study selected stage III hepatocellular carcinoma as samples for follow-up research.

Traditional Chinese medicine (TCM) refers to medicines used in China for thousands of years; they are typically derived from different natural medicines and herbal products and have made significant contributions to human health (Tao et al., 2015; Hao et al., 2017; Cheng et al., 2019; Xiao-Cong et al., 2018). Advances in analytical technologies and methodologies have accelerated the research of TCM, and the role of TCM in anti-tumor alternative therapies has received increasing attention (Lee et al., 2019). Studies have confirmed that TCM is effective in improving the survival rate of patients with breast and lung cancers (Lee et al., 2014; Hsiao \& Liu, 2010; Liao et al., 2017). SiNiSan (SNS) is a basic TCM formulation and was first introduced in the Treatise on Febrile Disease by Zhang Zhongjing, a famous physician in the late eastern Han Dynasty. SNS consists of 4 herbs: Gancao (Radix Glycyrrhizae), Chaihu (Radix Bupleuri), Zhishi (Fructus Aurantii Immaturus), and Baishao (Radix Paeoniae Alba). It is used for treating liver stagnation and spleen deficiency, improving disorders of the digestive system, and alleviating depression (Jiang et al., 2003). Studies have confirmed that SNS can affect the invasiveness and metastatic potential of hepatocellular carcinoma cells by inhibiting the phosphorylation of extracellular signal-related kinases and c-Jun N-terminal kinase signaling pathway (Hung-Jen et al., 2015). The mechanism is unclear, which has limited its clinical applications, thus, the therapeutic effects of SNS on hepatocellular carcinoma needs to be determined. Network pharmacology (NP) is an emerging drug research strategy that places the effects of drugs on diseases in a complex biological network and uses related gene databases to determine the mechanism of action of the drug compounds (Zhang et al., 2019). NP has accelerated pharmacological development and improved our understanding of mechanisms of drug action by establishing a multi-layer network of disease-phenotype-gene-drug (Xiao-Ming \& Chun-Fu, 2015) and is an important application for TCM research.

We downloaded the active pharmaceutical ingredients and target genes of SNS from the TCMSP database and obtained the disease genes of stage III hepatocellular carcinoma (HCC) from the TCGA and GEO databases. Gene Set Enrichment Analysis (GSEA) enrichment analysis was conducted on the gene sets of the two databases, and differential analysis was conducted to screen out the HCC genes with significant differences. The compound-disease target network was obtained from the intersection of the SNS targets and the HCC differential 
120 genes. The possible mechanisms and pathways of SNS were studied by analyzing the active

121 compounds of SNS in the network, performing GO and KEGG enrichment analysis on the

122 intersected genes, and constructing a protein interaction network. Finally, we used molecular

123 docking to simulate the process of SNS active molecules acting on target HCC genes. We

124 investigated the bioactive compounds, crossing targets, and possible mechanisms of SNS on

125 hepatocellular carcinoma using a network pharmacology strategy to provide new theoretical

126 treatments.

127

128 Materials \& Methods

129 Screening of bioactive compounds and targets in SiNiSan

130 The individual herbs and active components (Radix Glycyrrhizae, Radix Bupleuri, Fructus

131 Aurantii Immaturus and Radix Paeoniae Alba) in SNS were mined from the Traditional Chinese

132 Medicine Systems Pharmacology Database (TCMSP: http://tcmspw.com/tcmsp.php)/. This

133 database contains the active ingredients of Chinese medicine and their corresponding targets

134 (Yang et al., 2019). Herbal medicines are screened by TCMSP based on absorption, distribution,

135 metabolism, and excretion (ADME) and includes factors like oral bioavailability (OB), drug-

136 likeness (DL), and P450 (Lee et al., 2019). OB represents the speed and degree of drug

137 absorption in the circulation and DL represents the similarity between the herbal ingredients and

138 specific medicines, suggesting that herbs may be used as therapeutic agents. Refer to TCMSP's

139 platform research and related traditional Chinese medicine research in the past (Jinlong et al.,

140 2014; Xue et al., 2012), we selected OB and DL as conditions and set the filter criteria to

$141 \mathrm{OB} \geq 30 \%$ and $\mathrm{DL} \geq 0.18$ for potential bioactive compounds by Strawberry-perl software

142 (https://www.perl.org, ver.5.30.1.1).

143 Prediction and Gene Set Enrichment Analysis (GSEA) enrichment analysis of stage III

144 hepatocellular carcinoma targets

145 HCC-associated targets were acquired from The Cancer Genome Atlas (TCGA) and Gene

146 Expression Omnibus (GEO). TCGA is a collaborative database from the National Cancer

147 Institute (NCI) and the National Human Genome Institute (NHGRI), which is responsible for a

148 large amount of clinical and genomic data for various cancer types. The GEO database is an

149 international public gene expression database affiliated with NCBI that covers sufficient disease-

Peer) reviewing PDF | (2020:04:48251:2:1:NEW 28 Nov 2020) 
150 differential genes profiling by array (Barrett et al., 2013). TCGA data were obtained from the 151 GDC Data Portal (https://portal.gdc.cancer.gov/), select the TCGA-LIHC (Liver Hepatocellular

152 Carcinoma) project in the liver cancer classification, pick and summarize the stage III

153 hepatocellular carcinoma cases as the disease Group, and the normal adjacent tissues in HCC

154 cases as the normal group. Similarly, search for HCC data in the GEO database

155 (https://www.ncbi.nlm.nih.gov/) with "hepatocellular carcinoma" and "normal" as keywords, and 156 set the conditions as Homo sapiens to obtain a set of HCC gene expression profiles

157 (GSE101685) and its RNA chip annotation files (GPL570), then based on the annotation file, use 158 Strawberry-perl-5.30.1.1 software to convert the chip probe ID into gene symbol, and selected 159 the HCC samples of stage III as the disease group, and the normal tissue samples as the normal 160 group. GSEA calculated the results of the overall pathway or function enrichment through 161 enrichment analysis of the gene sets that did not undergo differential analysis nor did it interfere with artificially set thresholds, which is to determine whether the predefined gene set can show a consistent enrichment trend in overall genes include the disease group and the normal group. In order to perform GSEA analysis, the above-mentioned GEO and TCGA gene data were sorted into a matrix file which includes the disease group and the normal group. We selected the Canonical pathways of the C2 curated gene sets (c2.cp.pid.v7.1) in the Molecular Signatures Database (MSigDB) and analyzed them using GSEA software (ver 4.0.3).

Differential analysis of stage III hepatocellular carcinoma targets and the building of a compound-disease target network

Analyzing the HCC sample genes and normal sample genes from TCGA and GEO, and get the differentially expressed genes (DEGs) that are significantly different from the normal group in the disease group. DEGs for stage III HCC were obtained by limma package in R software (https://www.r-project.org, ver 3.6.2) based on two criteria $(|\operatorname{logFC}|>1$ and adj.P Value $<0.05)$.

174 The duplicates were removed after obtaining the differential genes from the TCGA and GEO databases. We determined the intersection of the SNS bioactive drug targets and DEGs of HCC using Strawberry-perl-5.30.1.1 software to obtain the targets for compound-disease network. The compound-disease target network was built, visualized, and analyzed using Cytoscape software

178 (https://cytoscape.org, ver3.7.2). The nodes in the network indicated the HCC target genes and 179 the SNS bioactive compounds. The edges indicated their interactions. 
181 The protein-protein interaction (PPI) network was constructed using the string database 182 (https://string-db.org/) based on crossing targets that determined the interactions between gene 183 regulatory proteins. The string database contains a large amount of protein interaction 184 information from experiments or other databases and can predict the mode of action between 185 HCC-related proteins. We entered 64 intersection HCC genes, selected the species as Homo 186 sapiens, and set the minimum required interaction score to 0.4 (default) to set up a PPI network.

187 The active protein interaction sources included text mining, experiments, databases, co188 expressions, neighborhoods, gene fusions, and co-occurrence. We imported the network to 189 Cytoscape for node and connection analyses.

190 GO and KEGG pathway enrichment analysis

191 We conducted Gene Ontology (GO) and Kyoto Encyclopedia of Genes and Genomes (KEGG) 192 enrichment analysis of 64 HCC target genes to clarify the biological functions and pathways of 193 SNS compounds for treating HCC. GO enrichment analysis included the biological process (BP) 194 and cell compounds (CC). GO and KEGG enrichment analysis $(\mathrm{P} \leq 0.05, \mathrm{Q} \leq 0.05)$ were conducted 195 using the bioconductor package in R software (ver3.6.2).

196 Molecular docking to simulate the core target-compound binding

197 We downloaded the PDB file of targets from the RCSB PDB website 198 (https://www.rcsb.org/\#Category-search) and the MOL2 file of the compound ligand from the 199 ZINC database (http://zinc.docking.org/) and selected a target related to HCC and performed 200 molecular docking with the corresponding compound. We used the AutoDock software 201 (ver4.2.6) to dehydrate and hydrogenate the macromolecules. We calculated the charge of the 202 target macromolecular protein, determined the rotation bond of the ligand, selected the 203 macromolecule, set the box, ran Autogrid to make the macromolecular crystal structure, and ran 204 Autodock to create a rigid docking of the macromolecules and ligands. The docking algorithm 205 was set as the local search parameter using Python 2.5 and we used Pymol software (ver2.3.4) to 206 perform the visualization of the docking result.

\section{Results}

\section{Bioactive compounds and targets in SiNiSan}


210 A total of 779 compounds and 6984 targets in SNS were collected from TCMSP. We obtained 21192 bioactive compounds and 1769 targets in Radix Glycyrrhizae, 17 bioactive compounds and 212123 targets in Radix Bupleur, 22 bioactive compounds and 348 targets in Fructus Aurantii 213 Immaturus, 13 bioactive compounds and 306 targets in Radix Paeoniae Alba with 21 repeat 214 compounds after screening by $\mathrm{OB} \geq 30 \%$ and $\mathrm{DL} \geq 0.18$. 113 compounds were identified as 215 potential bioactive molecules for further study.

\section{Hepatocellular carcinoma-associated targets and GSEA enrichment analysis results}

217 The genetic data from the TCGA database included 47 stage III hepatocellular tumor samples 218 and 7 and normal adjacent tissue samples. The dataset (GSE101685) selected from the GEO 219 database had 24 samples including 8 control (normal) samples and 16 test (stage III HCC) 220 samples. The results of GSEA enrichment analysis of the HCC gene sets in the two databases 221 showed that the stage III hepatocellular carcinoma group from TCGA was significantly enriched 222 in 82 pathways (nominal $\mathrm{p}$ value $<0.05$ and FDR $<25 \%$ ), sorted by their normalized enrichment 223 score (NES). The pathways with the highest enrichment levels are shown in Figure 1. The stage 224 III hepatocellular carcinoma group from GEO was significantly enriched in 9 pathways (nominal $225 \mathrm{p}$ value $<0.05$ and FDR $<25 \%$ ), as shown in Figure 2. The enrichment bar plot of the genes in the 226 two databases are shown in Figure 3. Based on the above results, all 9 pathways enriched in the HCC gene set from GEO are also enriched in the TCGA data. HCC genes were significantly enriched in the ATR, FANCONI, AURORA_B, PLK1, ARF3, MTOR4, P53_REGULATION, LKB1, ATM, and E2F pathways. All of these pathways are upregulated in HCC.

2569 differential genes were obtained after differential analysis including 1614 up-regulated genes and 955 down-regulated genes from TCGA. We obtained 1078 differential genes, including 370 up-regulated genes and 708 down-regulated genes from GEO. Differential expression genes of TCGA and GEO are shown in a volcano map (Figure 4).

\section{Analysis of compound-disease target network}

A total of $3101 \mathrm{HCC}$ differential genes were obtained after synthesizing the differential genes of the two databases and removing the duplicates. $64 \mathrm{HCC}$ target genes were obtained through the intersection of SNS targets and HCC differential genes. We built a visualized compound-disease target network by combining these with 113 bioactive compounds that had been previously obtained (Figure 5). 177 nodes and 610 edges are shown in the network. Most SNS molecules 
24140 edges, followed by kaempferol (MOL000422) with 17 edges. Table 1 shows the 30 most

242 prevalent ingredients in SNS prescriptions that act on the largest number of HCC targets. PTGS2

243 (106 edges), ESR1 (84 edges), AR (74 edges), NOS2 (74 edges), CCNA2 (53 edges) and

244 CHEK1 (50 edges) are the genes regulated by at least 1 compound. The target gene linkage

245 is $>50$ degrees, indicating that these targets might be a key target for SNS treatment of HCC.

246 Analysis of protein-protein interactions (PPI) network

247 The protein interactions of HCC differential genes extracted from the human genome were

248 shown in the PPI network (Figure 6). Each node in the network diagram represents a target gene,

249 the connection between the nodes indicates that there is an interaction relationship, and the color

250 of the nodes changing from blue to red represents the degree of the nodes changing from low to

251 high. The overall network included 64 nodes and 343 edges (PPI enrichment p-value: <1.0e-16),

252 the average node degree was 10.7 and the genes with the highest node degrees are: ESR1 (31

253 edges), MYC (30 edges), JUN (30 edges), FOS (25 edges), CAT (24 edges), and AR (22 edges).

254 GO and KEGG pathway enrichment analysis

255 The results of GO enrichment analysis of the $64 \mathrm{HCC}$ target genes showed that the effect of SNS

256 on HCC may be mainly associated with the response to factors including metalion, cadmiumion,

257 oxidative stress, steroid hormone, histone phosphorylation, cyclin-dependent protein kinase

258 holoenzyme complex, RNA polymerase II transcription factor complex, and the chromosomal

259 region. The distribution of enrichment results is shown in Figure 7. According to KEGG

260 enrichment analysis of HCC target genes, the major pathways for herbal interventions in HCC

261 are the p53 signaling pathway (hsa04115), calcium signaling pathway (hsa04020), NF-kappa B

262 signaling pathway (hsa04064), and the HIF-1 signaling pathway (hsa04066). The enrichment

263 results of KEGG pathways are shown in Table 2.

264 Docking results of compound and core target

265 Our results showed that quercetin can act on the greatest number of HCC targets. Therefore, we 266 docked the core targets involved in the component disease network (AR, BAX, CHEK2, ESR1, 267 NOS2, PTGS2) with quercetin (Figure 5). We downloaded the PDB files of the HCC targets and 268 the Mol2 files of the ligand quercetin (ZINC6484693) for docking. The quercetin structure has 6 269 rotatable bonds and the hydrogen bond binding energy, total internal energy, and electrostatic 270 energy are distinct due to its position and the different positions of the amino acid fragments of 271 the target protein. The docking results of the quercetin ligand, the HCC target macromolecule, 
272 the hydrogen bond binding energy, total internal energy, and electrostatic energy are shown in

273 Table 3. The inhibition constant is the dissociation constant of the protein-inhibitor complex and

274 the inhibition constant is smaller as the inhibition strength increases. The molecular docking of

275 CHEK2, BAX, AR, PTGS2 and quercetin are shown in Figure 8.

276

\section{Discussion}

278 HCC arises from hepatocytes in the liver parenchyma and is typically seen in patients with 279 chronic liver disease, including viral hepatitis, alcoholic liver disease, and nonalcoholic fatty 280 liver disease (Arslanoglu et al., 2016). Hepatocarcinogenesis is a multi-factorial process with 281 various signaling pathways that include the p53 signaling pathway, VEGF signaling pathway, 282 TGF signaling pathway, and Ras MARK signaling pathway (Grandhi et al., 2016). 75\% of 283 patients with limited stage cancer have a survival rate of approximately 5 years when treated 284 with curative therapies such as liver transplantation and surgical resection (Guy et al., 2012). 285 However, for patients in hepatic decompensation or with a highly heterogeneous tumor, surgery 286 is not always effective and has a significant economic burden on patients. HCC is resistant to 287 chemotherapy, thus an alternative treatment must be established.

288 TCM is prepared according to the principle of "King, Vassal, Assistant, and Delivery servant" 289 based on the patient's disease state (Zhang et al., 2019). This principle determines the major 290 therapeutic compounds combined with other agents used to restore the balance of body functions 291 and reduce toxicity, which can be exhibited in symptoms including insomnia, palpitation, 292 infections, and fever (Li et al., 2020). Studies have found that TCM plays an anti-tumor role 293 through apoptotic pathways in ROS-mediated mechanisms, DNA damage-mediated mechanisms, 294 Ca2+-mediated mechanisms, and by blocking transcription or translation (Min, 2010). TCM 295 inhibits cell proliferation and promotes the apoptosis of tumor cells by targeting the stimulation 296 of the host immune response for cytotoxic activity (Liao et al., 2015; Zhao et al., 2018). SNS has

297 been used for centuries in China as a remedy for liver stagnation and spleen deficiencies. Recent 298 experiments have shown that SNS is a remarkably effective treatment for hepatitis and liver 299 injury (Shu et al., 2018). The individual herbs that make up SNS have anti-inflammatory, anti300 bacterial, anti-oxidation, anti-tumor, anti-proliferation, anti-angiogenic, anti-HBV, and apoptotic 301 effects in vitro and in vivo (Zhao et al., 2018; Li et al., 2020; Ma et al., 2014; Sun et al., 2019; 302 Xiang et al., 2019; Pan et al., 2019); Zhou et al., 2019; Lefaki et al., 2020). 
303

304

305

306

307

308

309

310

311

312

313

314

315

316

317

318

319

320

321

322

323

324

325

326

327

328

329

330

331

332

333

In our research, there are 64 overlaps between drug targets and disease targets which are the important targets involved in the therapeutic mechanism of SNS, we studied 113 compounds and 64 target genes using the screening criteria and found that each bioactive compound acts on at least 1 HCC differential gene, core targets genes were PTGS2, ESR1, AR , NOS2, CCNA2 and CHEK1. The majority of the compounds were derived from Radix Glycyrrhizae (Gancao), which indicated that Radix Glycyrrhizae may be the principal active herb in treating HCC. Radix Bupleuri, Fructus Aurantii Immaturus, and Radix Paeoniae Alba may play a supporting role. Quercetin and kaempferol are bioactive ingredients with the highest number of targets and both coexist in multi-herb formulations (quercetin from Radix Glycyrrhizae and Radix Bupleuri, kaempferol from Radix Glycyrrhizae, Radix Bupleuri and Radix Paeoniae Alba). In order to study the molecular mechanism of disease development from a systematic perspective, we constructed a PPI network to predict the mode of action between HCC-related proteins and to provide a reference for the role of drugs. AR and ESR1 were found to be the targets that interact with the most proteins in the PPI network and also act as targets for a large number of drug components. This suggests that AR and ESR1 may be important nodes for SNS to act on HCC. AR is an androgen receptor involved in the growth and progression of hepatocellular carcinoma, it can inhibit the expression of CD90 in circulating tumor cells by up-regulating histone $3 \mathrm{H} 2 \mathrm{~A}$ (Han et al., 2020). Targeting the AR transduction pathway may be the key to inhibiting the progression of advanced HCC (Han et al., 2020; Ma et al., 2014). ESR1 is an estrogen receptor that causes abnormal serum methylation and can be used as a biomarker for the diagnosis of HCC. The imbalance of ESR1 expression can cause a high degree of HCC amplification (Tsiambas et al., 2011; Dou at al 2016) and is a focus of targeted therapy.

Enrichment analysis revealed that these compounds may act against HCC through: the cellular response to steroid hormones; oxidative stress; histone phosphorylation; steroid metabolic processes; cyclin-dependent protein kinase holoenzyme complex; synthesis of RNA polymerase II transcription factor complex and the nuclear transcription factor complex. The response of these biological functions on steroid hormones coincided with the target AR and ESR1, suggesting that SNS may regulate the levels of sex hormones. Histone H3 phosphorylation is involved in the chemical carcinogenesis of HCC, while histone 4 phosphorylation is related to the proliferation and differentiation of hepatocytes after a liver injury (Sukumar et al., 2020; Liu \& Liu, 2020). The potential pathways are: the p53 signaling 
334 pathway; calcium signaling pathway; NF-kappa B signaling pathway; and the HIF-1 signaling 335 pathway. The P53 pathway has the highest degree of enrichment, combined with the previous

336

337

338

339

340

341

342

343

344

345

346

347

348

349

350

351

352

353

354

355

356

357

358

359

360

361

362

363 gene set GSEA analysis. The P53 pathway is also significantly enriched in the overall stage III hepatocellular carcinoma gene set. Previous studies have demonstrated that P53 inhibits the progression, recurrence, and related immune responses of HCC (G et al., 2013; Xiaonian et al., 2017). The signaling pathway exists in p53 in HCC based on the HCC map in KEGG (https://www.kegg.jp) and acts as tumor suppressor. The SNS target genes in the p53 pathway include BAX, CDK1, CHEK1, CCNB1, SERPINE1, CHEK2, and IGFBP3. Quercetin interferes with 6 of the 7 target genes in the above-mentioned p53 pathway (BAX, CDK1, CCNB1, SERPINE1, CHEK2, IGFBP3) and molecular docking confirmed that quercetin can bind to the P53 pathwayrelated targets (CHEK2, SPRPINE1, BAX). Thus, quercetin is likely to be a promising HCC treatment drug in SNS. Quercetin is a bioflavonoid with high antitumor activity that acts on cancers such as osteosarcoma and colon cancer by rebuilding the tumor microenvironment and alleviates liver damage by regulating the EGFR pathway (Choi et al., 2010; Lan et al., 2017; Hu et al., 2017; Carrasco-Torres et al., 2017; Massi et al., 2017), Quercetin can exert anti-tumor activity by targeting HSP70/90, P13K, and NF-B and has been reported in the treatment of hepatocellular carcinoma (Fernández, F.P et al., 2019; Wang R. E et al 2011 ). Our other derived pathways require testing to determine their efficacy in the treatment of HCC.

\section{Conclusions}

TCM assumes the characteristics of multiple compounds, targets and pathways when applied to disease prevention and treatment, which is the focus of network pharmacology. We predicted the targets and potential mechanisms of SNS compounds for HCC treatment by constructing networks and used molecular docking to simulate their behavior. We focused on quercetin's targeting of genes that were enriched in the p53 pathway and then inhibited the progression of HCC by regulating the p53 pathway. GSEA enrichment analysis of the HCC gene set and the KEGG enrichment analysis of the intersection gene after the differential analysis show that the P53 pathway is a pathway with significant influence. AR and ESR1 in HCC targets are also worthy of attention due to their large number of SNS components and the proteins that are highly interactive with other proteins in the PPI network. Research has revealed that these two sex 
364 hormone receptors are involved in the development of HCC. SNS can inhibit HCC by affecting 365 hormone levels and by regulating the P53 pathway. The results of this study should be verified 366 by in vivo and vitro experiments and more studies are needed to reduce the toxicity of TCM and 367 prove its value in the treatment of HCC.

368

369

\section{Acknowledgements}

370 We would like to thank Professor Helin Feng for the research guidance and technical support of 371 this study.

372

373 References

374 Arslanoglu, A., Seyal, A.R., Sodagari, F., Sahin, A., Miller, F.H., Salem, R., and Yaghmai, V. 375 2016. Current Guidelines for the Diagnosis and Management of Hepatocellular Carcinoma: A 376 Comparative Review. AJR. American journal of roentgenology 207:W88.

377 Barrett, T., Wilhite, S.E., and Ledoux, P. 2013. NCBI GEO: archive for functional genomics data 378 sets--update. NUCLEIC ACIDS RESEARCH 41.

379 Carrasco-Torres, G., Monroy-Ramírez, H.C., Martínez-Guerra, A.A., Baltiérrez-Hoyos, R., 380 Romero-Tlalolini, M.D.L.Á., Villa-Treviño, S., Sánchez-Chino, X., and Vásquez-Garzón, V.R. 381 2017. Quercetin Reverses Rat Liver Preneoplastic Lesions Induced by Chemical Carcinogenesis. 382 Oxidative Medicine and Cellular Longevity 2017. 10.1155/2017/4674918

383 Cheng, X., Wence, Z., Yuming, W., and Liang, Q. 2014. Hepatitis B virus-induced

384 hepatocellular carcinoma. Cancer Letters 345:216-222.

385 CHENG, X.R., QI, C.H., and WANG, T.X. 2019. Characteristics of the traditional Liu-Wei-Di386 Huang prescription reassessed in modern pharmacology. Chinese Journal of Natural Medicines $387 \quad 17: 103-121$.

388 Choi, K.C., Chung, W.T., Kwon, J.K., Yu, J.Y., Jang, Y.S., Park, S.M., Lee, S.Y., and Lee, J.C. 389 2010. Inhibitory effects of quercetin on aflatoxin B1-induced hepatic damage in mice. FOOD 390 AND CHEMICAL TOXICOLOGY 48:2747-2753. 10.1016/j.fct.2010.07.001 
391 Dou CY., Fan YC., Cao CJ., Yang Y., Wang K. 2016. Sera DNA Methylation of CDH1, 392 DNMT3b and ESR1 Promoters as Biomarker for the Early Diagnosis of Hepatitis B Virus393 Related Hepatocellular Carcinoma. Digestive Diseases and Sciences 61: 1130-1138.

394 10.1007/s10620-015-3975-3

395 Ferlay, J., Colombet, M., Soerjomataram, I., Mathers, C., Parkin, D.M., Piñeros, M., Znaor, A., 396 and Bray, F. 2019. Estimating the global cancer incidence and mortality in 2018: GLOBOCAN 397 sources and methods. INTERNATIONAL JOURNAL OF CANCER 144:1941-1953.

$398 \quad 10.1002 /$ ijc.31937

399 Forner, A., Reig, M., and Bruix, J. 2018. Hepatocellular carcinoma. LANCET 391:1301-1314.

400 G, P., Besant, P.V., and Attwood. 2013. Histone H4 histidine phosphorylation: kinases, 401 phosphatases, liver regeneration and cancer. BIOCHEMICAL SOCIETY TRANSACTIONS 402 40:290-293.

403 Grandhi, M.S., Kim, A.K., Ronnekleiv-Kelly, S.M., Kamel, I.R., Ghasebeh, M.A., and Pawlik, 404 T.M. 2016. Hepatocellular carcinoma: From diagnosis to treatment. Surgical Oncology 25:74-85. 405 10.1016/j.suronc.2016.03.002

406 Guy, J., Kelley, R.K., Roberts, J., Kerlan, R., Yao, F., and Terrault, N. 2012. Multidisciplinary 407 Management of Hepatocellular Carcinoma. Clinical Gastroenterology and Hepatology 10:354408 362. 10.1016/j.cgh.2011.11.008

409 Han Q., Yang D., Yin C., Zhang J. Androgen Receptor (AR)-TLR4 Crosstalk Mediates Gender 410 Disparities in Hepatocellular Carcinoma Incidence and Progression. Journal of Cancer 11: 1094411 1103. 10.7150/jca.30682

412 Hao, P., Jiang, F., Cheng, J., Ma, L., Zhang, Y., and Zhao, Y. 2017. Traditional Chinese 413 medication for cardiovascular disease. JOURNAL OF THE AMERICAN COLLEGE OF 414 CARDIOLOGY 69.

415 Hsiao, W.L.W., and Liu, L. 2010. The Role of Traditional Chinese Herbal Medicines in Cancer 416 Therapy - from TCM Theory to Mechanistic Insights. PLANTA MEDICA 76.

417 Hu, K., Miao, L., Goodwin, T.J., Li, J., Liu, Q., and Huang, L. 2017. Quercetin Remodels the 418 Tumor Microenvironment To Improve the Permeation, Retention, and Antitumor Effects of 419 Nanoparticles. ACS Nano 11:4916-4925. 10.1021/acsnano.7b01522 
420 Hung, J.L., Shung, T.K., Yu, M.S.,Chia, C.Y. 2015. The Chinese medicine Sini-San inhibits 421 HBx-induced migration and invasiveness of human hepatocellular carcinoma. BioMedCentral 422 Complementary and Alternative Medicine 15:348. 10.1186/1758-2946-6-13

423 Jiang, J., Zhou, C., Xu, Q., China, P.U., School, O.L.S., ADepartment, O.P.F.C., Nanjing, U., 424 and BState, K.L.O.P. 2003. Alleviating Effects of Si-Ni-San, a Traditional Chinese Prescription, 425 on Experimental Liver Injury and Its Mechanisms. Biological and Pharmaceutical Bulletin 426 26:1089-1094. 10.1248/bpb.26.1089

427 Jinlong, R., Peng, L, Jinan, W., Wei, Z., Bohui, L., Chao, H., Pidong, L., Zihu, G., Weiyang, T., 428 Yinfeng, Y., Xue, X., Yan, L., Yonghua, W., Ling Y. 2014. TCMSP: a database of systems 429 pharmacology for drug discovery from herbal medicines . Journal of Cheminformatics, 2014, 6: 43013.

431 Heimbach, JK., Kulik, LM., Finn, R., Sirlin, CB., Abecassis, M., Roberts, LR., Zhu, A., Murad, 432 MH., Marrero, J. 2018. AASLD Guidelines for the Treatment of Hepatocellular Carcinoma. 433 HEPATOLOGY 67: 358-380. 10.1002/hep.29086.

434 Kudo, M. 2011. Molecular Targeted Therapy for Hepatocellular Carcinoma: Bench to Bedside. 435 DIGESTIVE DISEASES-BASEL- 29.

436 Lan, H., Hong, W., Fan, P., Qian, D., Zhu, J., and Bai, B. 2017. Quercetin Inhibits Cell 437 Migration and Invasion in Human Osteosarcoma Cells. CELLULAR PHYSIOLOGY AND 438 BIOCHEMISTRY 43:553-567. 10.1159/000480528

439 Lee, W., Lee, C., Kim, Y., and Kim, C. 2019. The Methodological Trends of Traditional Herbal 440 Medicine Employing Network Pharmacology. Biomolecules 9:362. 10.3390/biom9080362

441 Lee, Y., Chen, T., Shih, Y.V., Tsai, C., Chang, C., Liang, H., Tseng, S., Chien, S., and Wang, C. 442 2014. Adjunctive Traditional Chinese Medicine Therapy Improves Survival in Patients With 443 Advanced Breast Cancer. CANCER 120:1338-1344. 10.1002/cncr.28579

444 Lefaki, M., Papaevgeniou, N., Tur, J.A., Vorgias, C.E., Sykiotis, G.P., and Chondrogianni, N. 445 2020. The dietary triterpenoid 18 $\alpha$-Glycyrrhetinic acid protects from MMC-induced 446 genotoxicity through the ERK/Nrf2 pathway. Redox Biology 28:101317.

447 10.1016/j.redox.2019.101317

448 Li, J., Tang, F., Li, R., Chen, Z., Lee, S.M., Fu, C., Zhang, J., and Leung, G.P. 2020. Dietary 449 compound glycyrrhetinic acid suppresses tumor angiogenesis and growth by modulating 
450 antiangiogenic and proapoptotic pathways in vitro and in vivo. The Journal of Nutritional 451 Biochemistry 77:108268. 10.1016/j.jnutbio.2019.108268

452 Li, L., Qiu, H., Liu, M., and Cai, Y. 2020. A Network Pharmacology-Based Study of the 453 Molecular Mechanisms of Shaoyao-Gancao Decoction in Treating Parkinson's Disease. 454 Interdisciplinary Sciences: Computational Life Sciences. 10.1007/s12539-020-00359-7

455 Liao, Y., Lin, C., Lai, H., Chiang, J., Lin, J., and Li, T. 2015. Adjunctive traditional Chinese 456 medicine therapy improves survival of liver cancer patients. LIVER INTERNATIONAL 35.

457 Liao, Y.H., Li, C.I., Lin, C.C., Lin, J.G., Chiang, J.H., and Li, T.C. 2017. Traditional Chinese 458 medicine as adjunctive therapy improves the long - term survival of lung cancer patients. Journal 459 of Cancer Research and Clinical Oncology 143:2425-2435. 10.1007/s00432-017-2491-6 460 Liu, X., and Liu, J. 2020. Tanshinone I induces cell apoptosis by reactive oxygen species461 mediated endoplasmic reticulum stress and by suppressing p53/DRAM-mediated autophagy in 462 human hepatocellular carcinoma. Artificial Cells, Nanomedicine, and Biotechnology 48:488-497. $46310.1080 / 21691401.2019 .1709862$

464 Ma, X., Jin, S., Zhang, Y., Wan, L., Zhao, Y., and Zhou, L. 2014. Inhibitory Effects of Nobiletin 465 on Hepatocellular CarcinomaIn Vitro andIn Vivo. PHYTOTHERAPY

466 RESEARCH 28:560-567. 10.1002/ptr.5024

467 Maheshkumar, K., Sridharan, J., Kannan, M.R., Chander, C.S., Mathan, G., and Velanganni, 468 A.A.J. 2019. AEG-1/miR-221 Axis Cooperatively Regulates the Progression of Hepatocellular 469 Carcinoma by Targeting PTEN/PI3K/AKT Signaling Pathway. INTERNATIONAL JOURNAL 470 OF MOLECULAR SCIENCES 20.

471 Massi, A., Bortolini, O., Ragno, D., Bernardi, T., Sacchetti, G., Tacchini, M., and De Risi, C. 472 2017. Research Progress in the Modification of Quercetin Leading to Anticancer Agents.

473 MOLECULES 22:1270. 10.3390/molecules22081270

474 Ma WL., Lai HC., Yeh S., Cai X., Chang C. 2014. Androgen receptor roles in hepatocellular 475 carcinoma, fatty liver, cirrhosis and hepatitis. Endocr. Relat. Cancer 21:R165-182 10.1530/ERC$476 \quad 13-0283$

477 Min, L. 2010. Targeting apoptosis pathways in cancer by Chinese medicine. CANCER LETTERS 478 2:304-312. 10.1016/j.canlet.2010.07.015

479 Pan, H., Fu, X., and Huang, W. 2011. Molecular mechanism of liver cancer. Anti-Cancer Agents 480 in Medicinal Chemistry 11:493-499. 
481 Pan, Y., Ke, Z., Ye, H., Sun, L., Ding, X., Shen, Y., Zhang, R., and Yuan, J. 2019. Saikosaponin

$482 \mathrm{C}$ exerts anti-HBV effects by attenuating HNF1 $\alpha$ and HNF4 $\alpha$ expression to suppress HBV

483 pgRNA synthesis. INFLAMMATION RESEARCH 68:1025-1034. 10.1007/s00011-019-01284-2

484 Fernández, F.P., Fondevila, F., Méndez, B. C.2019 Antitumor Effects of Quercetin in

485 Hepatocarcinoma In Vitro and In Vivo Models: A Systematic Review. Nutrient 11.

$48610.3390 /$ nu1 1122875

487 Sandra, R., and Jean-Charles, N. 2020. Advances in molecular classification and precision

488 oncology in hepatocellular carcinoma. JOURNAL OF HEPATOLOGY 72:215-229.

489 Shu, Z., He, W., Shahen, M., Guo, Z., Shu, J., Wu, T., Bian, X., Shar, A.H., Farag, M.R.,

490 Alagawany, M., and Liu, C. 2018. Clarifying of the potential mechanism of Sinisan formula for

491 treatment of chronic hepatitis by systems pharmacology method. BIOMEDICINE \&

492 PHARMACOTHERAPY 100:532-550. 10.1016/j.biopha.2018.02.047

493 Sukumar, U.K., Rajendran, J., Gambhir, S.S., Massoud, T.F., and Paulmurugan, R. 2020. SP94-

494 Targeted Triblock Co-Polymer Nanoparticle Delivers Thymidine Kinase-p53-Nitroreductase

495 Triple Therapeutic Gene and Restores Anticancer Function against Hepatocellular Carcinoma in 496 vivo. ACS Appl Mater Interfaces. 10.1021/acsami.9b20071

497 Sun, J., Liu, H., Lv, C., Qin, J., and Wu, Y. 2019. Modification, Antitumor Activity, and

498 Targeted PPAR $\gamma$ Study of 18ß-Glycyrrhetinic Acid, an Important Active Ingredient of Licorice.

499 JOURNAL OF AGRICULTURAL AND FOOD CHEMISTRY 67:9643-9651.

500 10.1021/acs.jafc.9b03442

501 Tao, W., Luo, X., Cui, B., Liang, D., Wang, C., Duan, Y., Li, X., Zhou, S., Zhao, M., Li, Y., He,

502 Y., Wang, S., Kelley, K.W., Jiang, P., and Liu, Q. 2015. Practice of traditional Chinese medicine

503 for psycho-behavioral intervention improves quality of life in cancer patients: A systematic

504 review and meta-analysis. Oncotarget 6:39725. 10.18632/oncotarget.5388

505 Tsiambas E., Georgiannos SN., Salemis N., Alexopoulou D., Lambropoulou S., Dimo B. 2011.

506 Significance of estrogen receptor 1 (ESR-1) gene imbalances in colon and hepatocellular

507 carcinomas based on tissue microarrays analysis. MEDICAL ONCOLOGY 28: 934-940.

508 10.1007/s12032-010-9554-8

509 Villanueva, A. 2019. Hepatocellular Carcinoma. The New England Journal of Medicine

510 380:1450-1462. 10.1056/NEJMra1713263 
511 Wang R. E., Hunt C. R.,Chen J, Taylor J. S. 2011. Biotinylated quercetin as an intrinsic

512 photoaffinity proteomics probe for the identification of quercetin target proteins. Bioorg. Med.

513 Chem. 19: 4710-20.

514 Xiang, Y., Zhang, Q., Wei, S., Huang, C., Li, Z., and Gao, Y. 2019. Paeoniflorin: a monoterpene 515 glycoside from plants of Paeoniaceae family with diverse anticancer activities. The Journal of 516 pharmacy and pharmacology. 10.1111/jphp.13204

517 Xiao-Cong, P., De, K., Jian-Song, F., and Ying, Z. 2018. Network pharmacology-based analysis 518 of Chinese herbal Naodesheng formula for application to Alzheimer's disease. Chinese Journal 519 of Natural Medicines 16:52-53.

520 Xiao-Ming, W., and Chun-Fu, W. 2015. Network pharmacology: A new approach to unveiling 521 Traditional Chinese Medicine. Chinese Journal of Natural Medicines 13:1-2.

522 Xiaonian, Z., Daochuan, L., Zhengbao, Z., Wei, Z., and Wenxue, L. 2017. Persistent

523 phosphorylation at specific H3 serine residues involved in chemical carcinogen-induced cell 524 transformation. MOLECULAR CARCINOGENESIS 56:1449-1460.

525 Xue X., Zhang W., Huang C., Li Y., Yu H., Wang Y., Duan J., Ling Y. 2012. A novel 526 chemometric method for the prediction of human oral bioavailability. International Journal of 527 Molecular Sciences 13:6964-6982. 10.3390/ijms13066964

528 Yang, H., Li, Y., Shen, S., Gan, D., Han, C., Wu, J., and Wang, Z. 2019. Network

529 Pharmacology-Based Investigation into the Mechanisms of Quyushengxin Formula for the 530 Treatment of Ulcerative Colitis. Evidence-based Complementary and Alternative Medicine 531 2019:22. 10.1155/2019/7870424

532 Zhang, R., Zhu, X., Bai, H., and Ning, K. 2019. Network Pharmacology Databases for

533 Traditional Chinese Medicine: Review and Assessment. Frontiers in Pharmacology 10.

534 10.3389/fphar.2019.00123

535 Zhao, S., Liu, Z., Wang, M., He, D., Liu, L., Shu, Y., Song, Z., Li, H., Liu, Y., and Lu, A. 2018.

536 Anti-inflammatory effects of Zhishi and Zhiqiao revealed by network pharmacology integrated 537 with molecular mechanism and metabolomics studies. PHYTOMEDICINE 50:61-72.

538 10.1016/j.phymed.2018.09.184

539 Zhou, F., Wu, G., Cai, D., Xu, B., Yan, M., Ma, T., Guo, W., Zhang, W., Huang, X., Jia, X.,

540 Yang, Y., Gao, F., Wang, P., and Lei, H. 2019. Synthesis and biological activity of 
541 glycyrrhetinic acid derivatives as antitumor agents. EUROPEAN JOURNAL OF MEDICINAL

542 CHEMISTRY 178:623-635. 10.1016/j.ejmech.2019.06.029

543

544

545 


\section{Figure 1}

The results of GSEA enrichment analysis of stage III HCC genes from TCGA database.

(A) The normalized enrichment score (NES) and significance analysis of C2 curated gene sets

(c2.cp.pid.v7.1) of GSEA; (B-I) The top 8 significantly enriched pathways ( $p$ value $<0.05$ and FDR $<25 \%$ ), namely: ARF pathway, ATM pathway, P53 REGULATION pathway, ATR pathway, AURORA A pathway, E2F pathway, FANCONI pathway and FOXM1 pathway. 
A

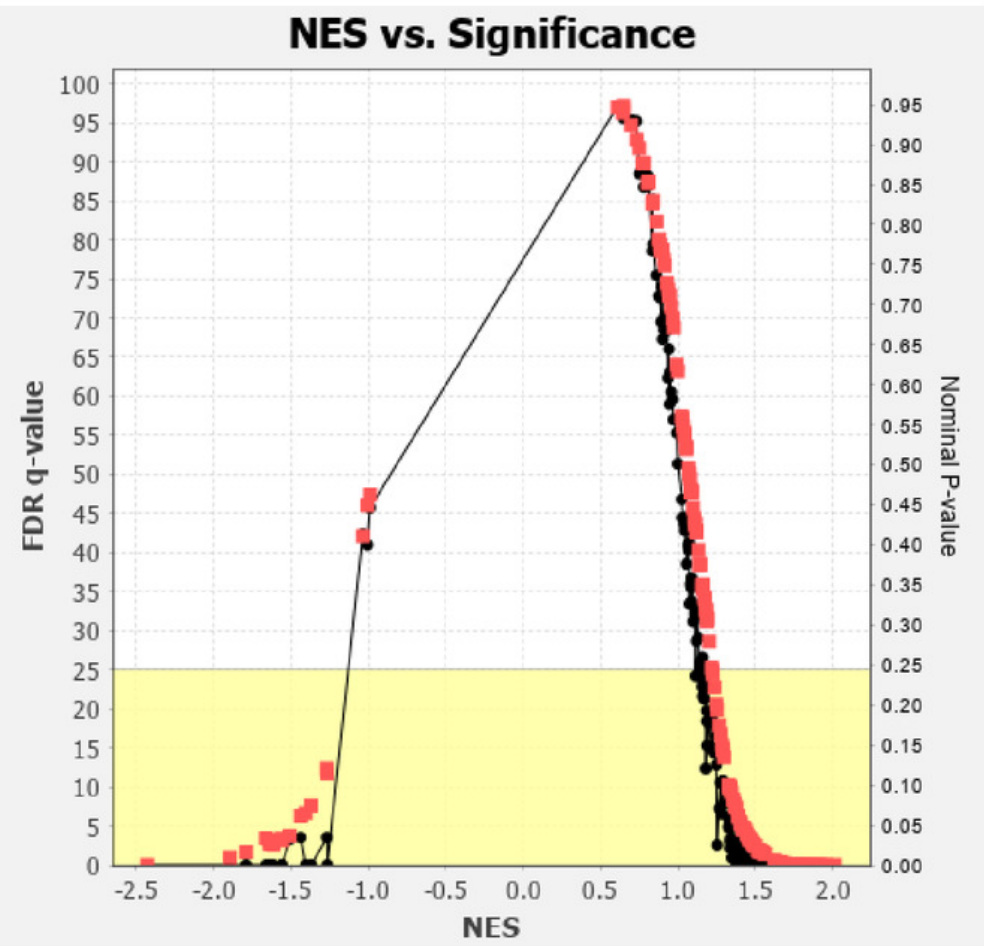

nDR q-value

D

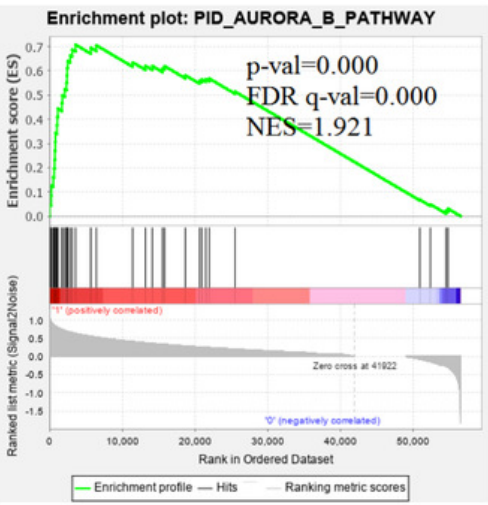

G

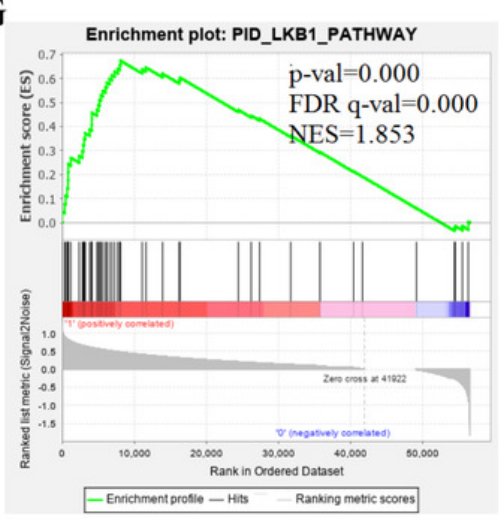

- nominal p-value

E

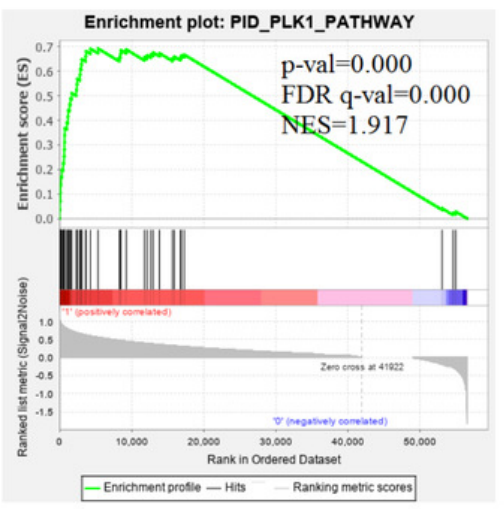

$\mathrm{H}$

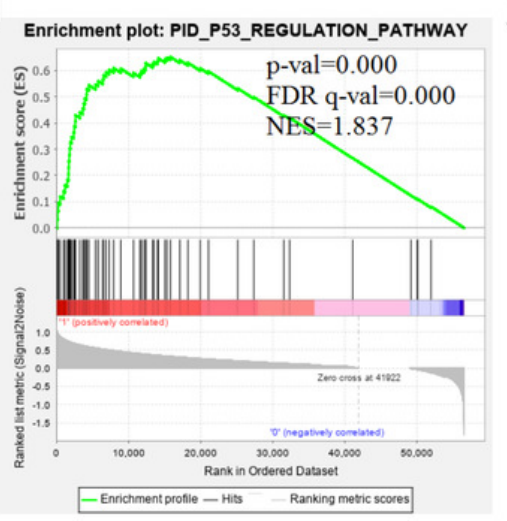

B

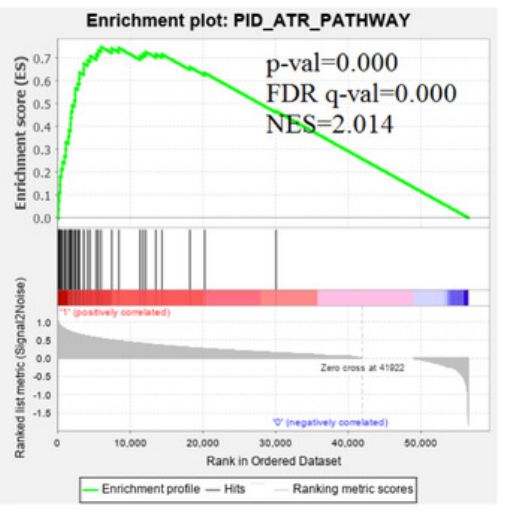

C

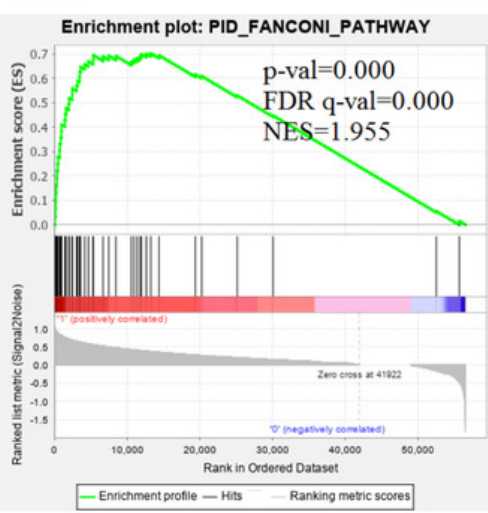

$\mathrm{F}$
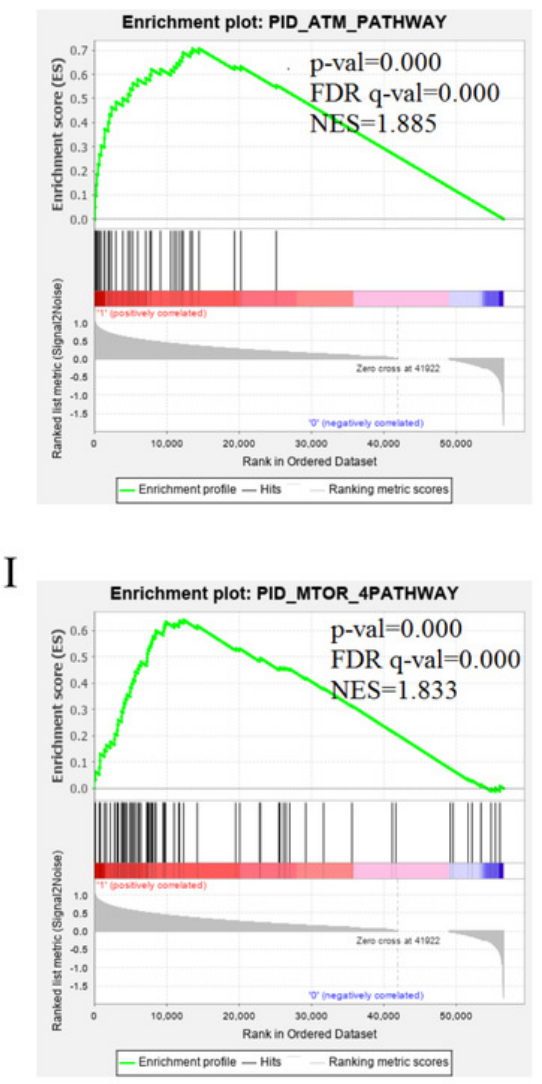
Figure 2

The results of GSEA enrichment analysis of stage III HCC genes from GEO database.

(A) The normalized enrichment score (NES) and significance analysis of C2 curated gene sets

(c2.cp.pid.v7.1) of GSEA; (B-I) The top 8 significantly enriched pathways ( $p$ value $<0.05$ and FDR<25\%), namely: ATR pathway, AURORA B pathway, DELTA NP63 pathway, FANCONI pathway, FOXM1 pathway, HIF1A pathway, P73 pathway and PLK1 pathway. 
A

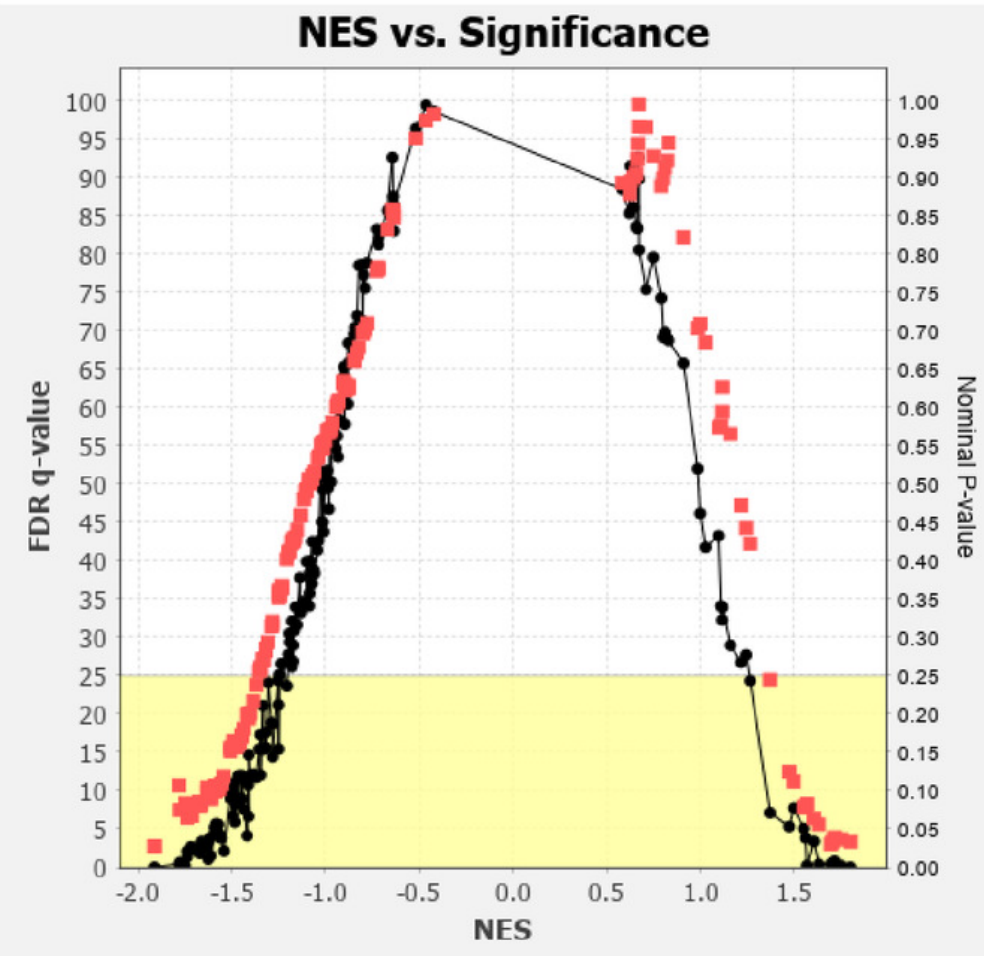

" FDR q-value

D

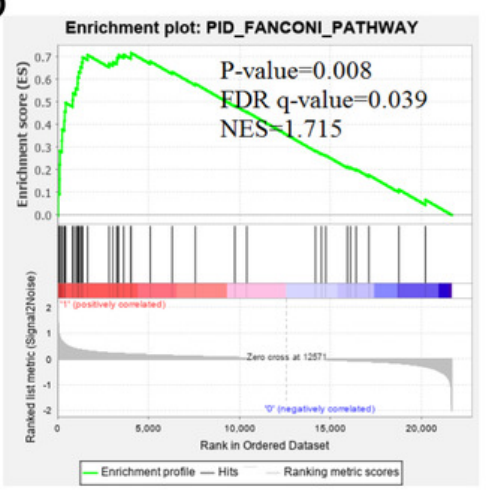

G Enrichment plot: PID_DELTA_NP63_PATHWAY

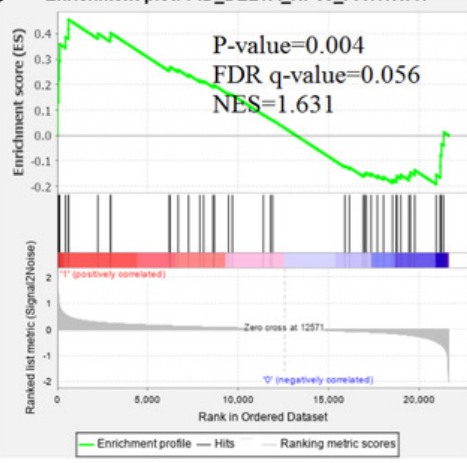

E

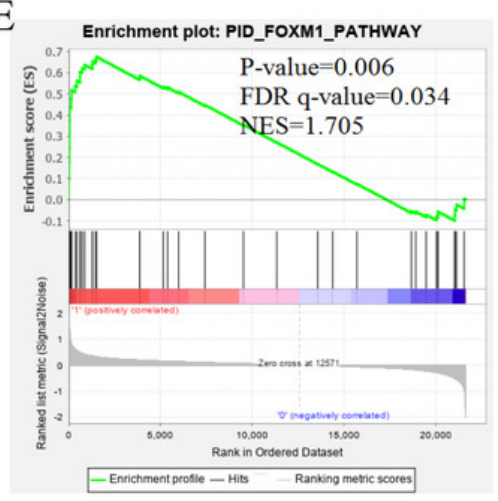

H

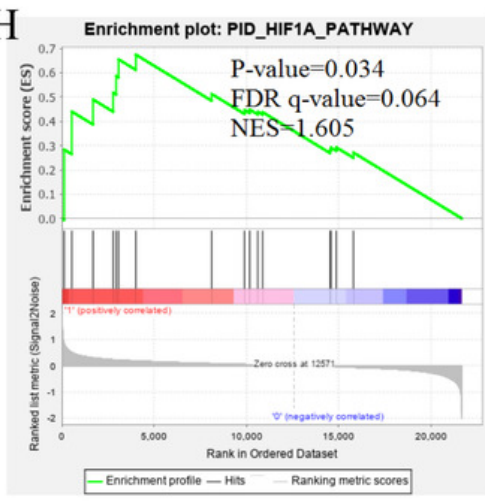

B

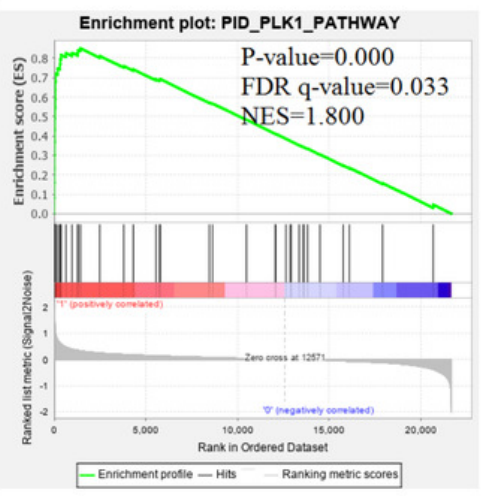

C

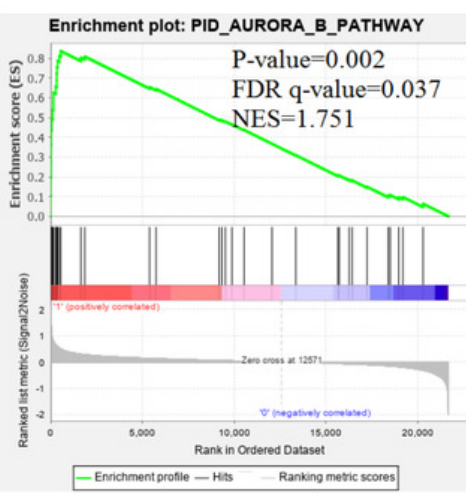

F

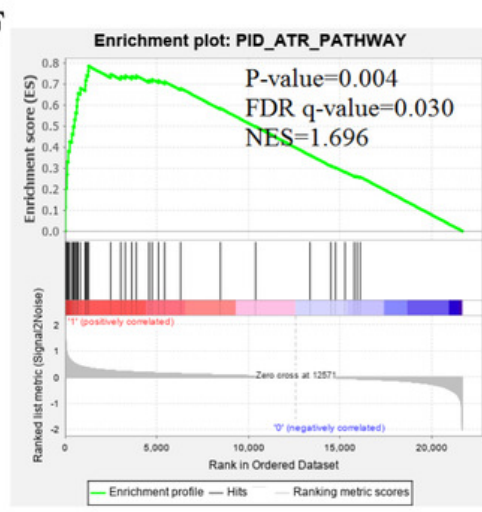

I

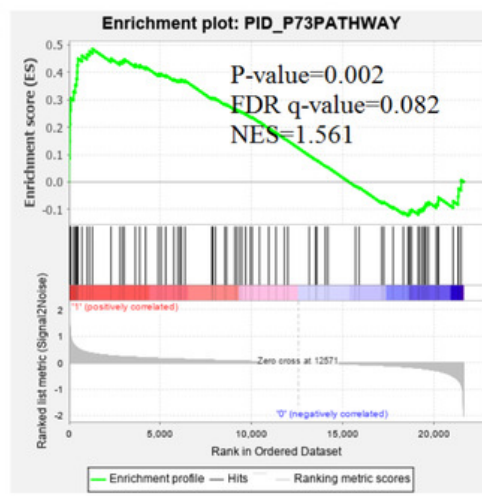


Figure 3

The enriched pathway of GSEA analysis based on HCC targets from TCGA and GEO database

(A) GSEA enrichment analysis of stage III HCC genes from TCGA database. (B) GSEA enrichment analysis of stage III HCC genes from GEO database.

A

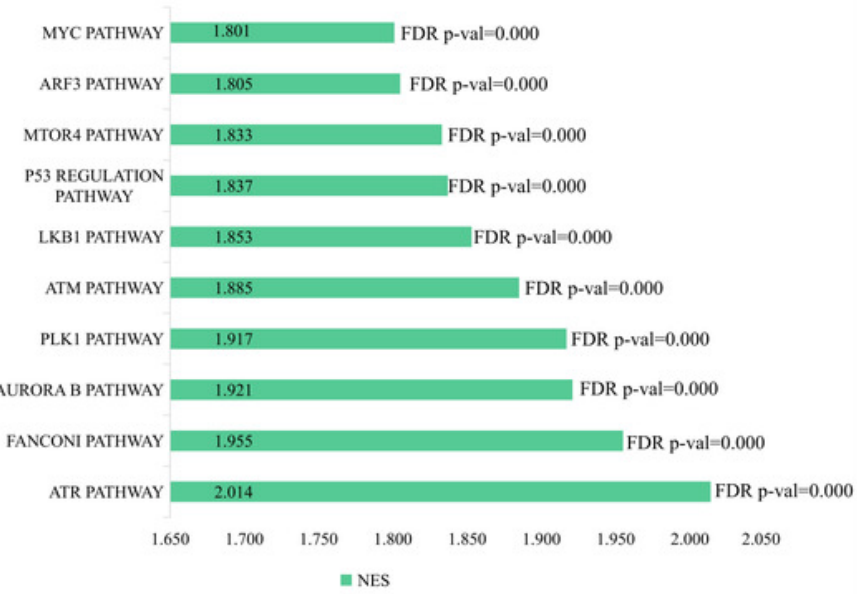

B

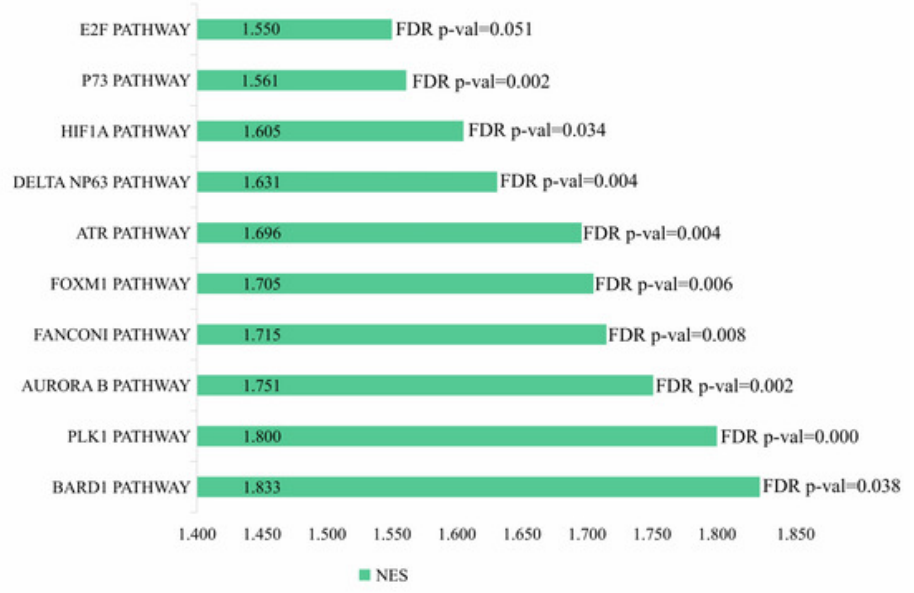


Figure 4

The difffferentially expressed genes(DEGs) between normal and stage III HCC tissues.

The top genes were marked (A) DEGs from TCGA database; (B) DEGs from GEO database.

$\mathbf{A}$

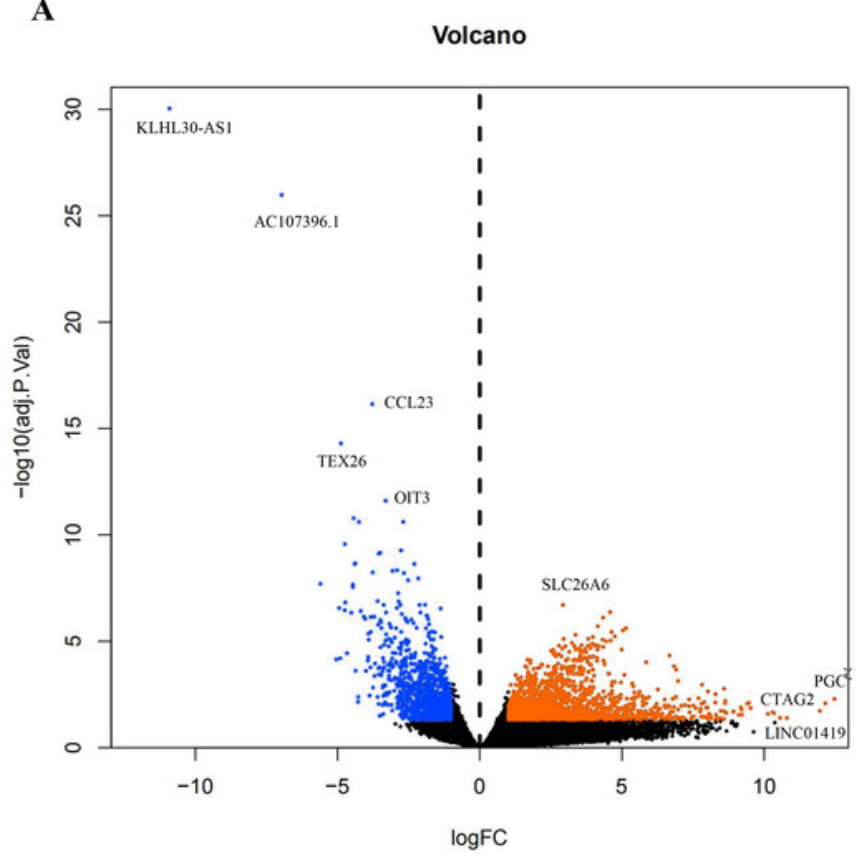

B

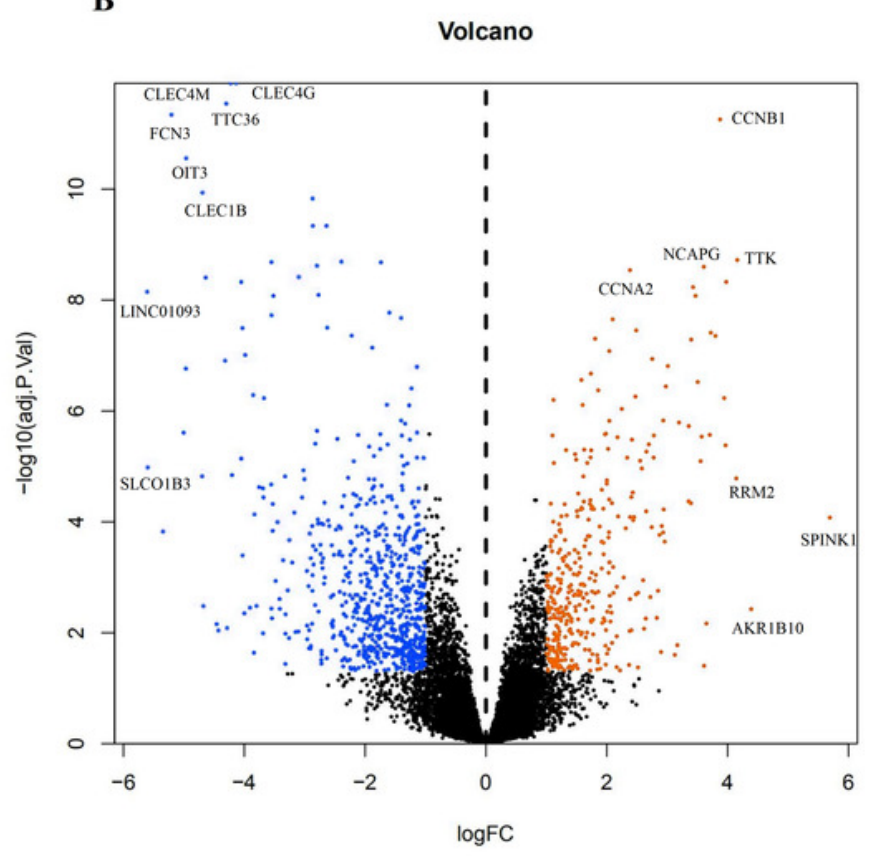




\section{Figure 5}

\section{The targets of each candidate compound in SiNiSan on stage III HCC.}

The outer circle represents the SNS component and the inner circle represents the HCC target, darkened nodes indicate key genes for drug targeting.

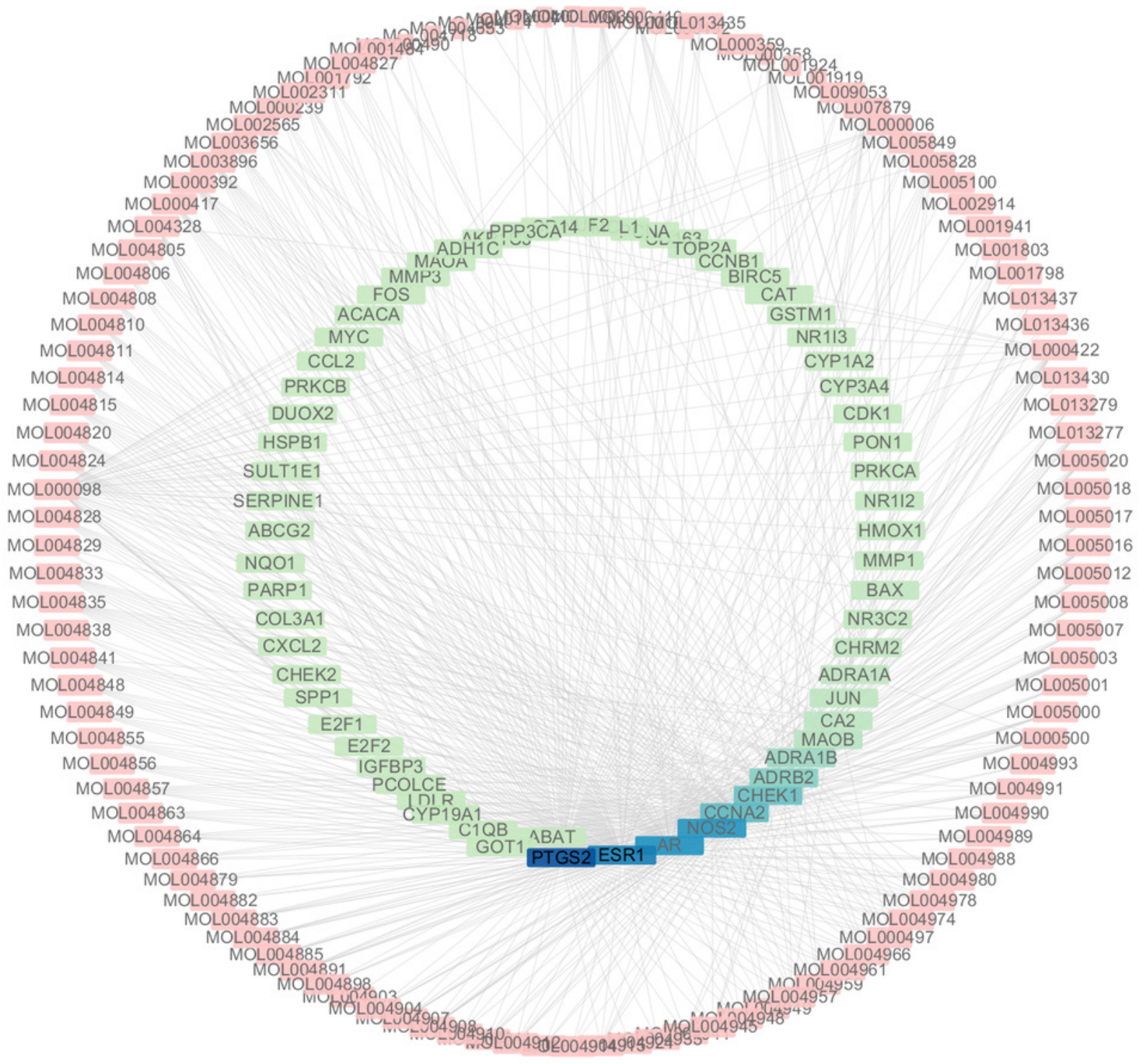




\section{Figure 6}

The PPI network of the 64 targets of SiNiSan on stage III HCC.

The color of the nodes changing from blue to red represents the degree of the nodes changing from low to high.

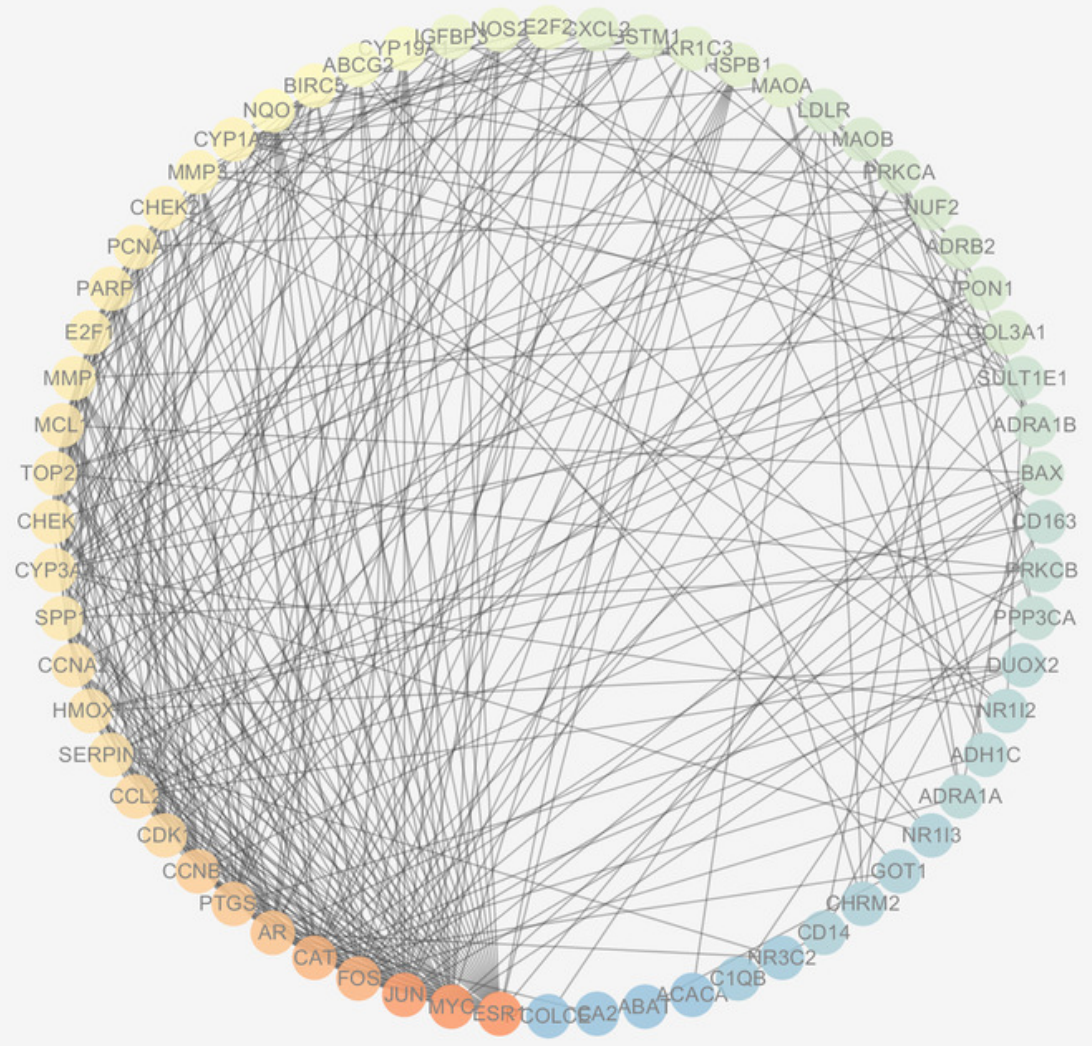


Figure 7

The results of GO enrichement analysis of the 64 targets of SiNiSan on stage III HCC.

(A) the top 20 biological processes (BPs); (B) the top 20 cell compounds (CCs).
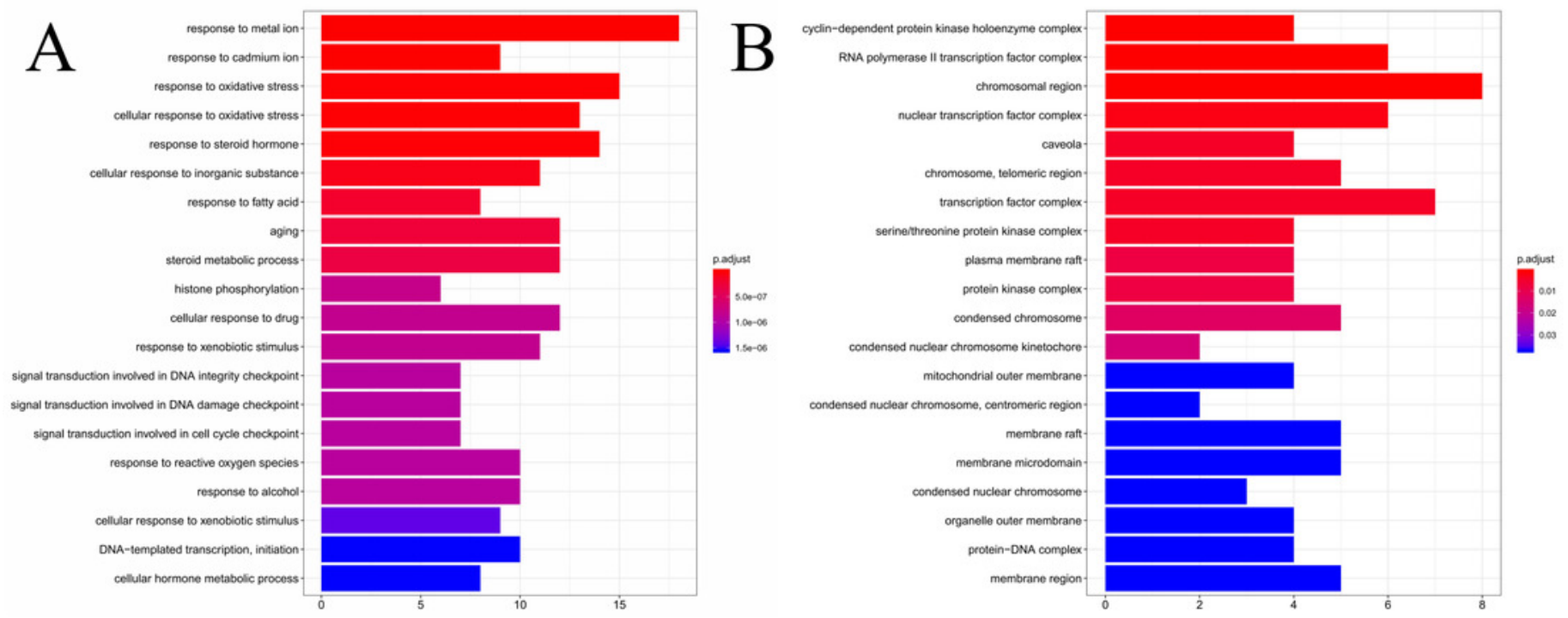


\section{Figure 8}

The results of molecular docking between quercetin and genes CHEK2, BAX, AR, PTGS2.

In the picture, the blue molecule is quercetin, the pink is the amino acid residue where the target protein is docked with quercetin, and the yellow dotted line is the hydrogen bond. (A)The molecular docking between quercetin ligand and CHEK2 macromolecule, the amino

acid residue forming a hydrogen bond with quercetin is ARG-482; (B)The molecular docking between quercetin ligand and BAX macromolecule, the amino acid residue forming a hydrogen bond with quercetin is LYS-64 and SER-60; (C)The molecular docking between quercetin ligand and AR macromolecule, the amino acid residue forming a hydrogen bond with quercetin is GLU-308; (D)The molecular docking between quercetin ligand and PTGS2 macromolecule, the amino acid residue forming a hydrogen bond with quercetin is ASN-43. 


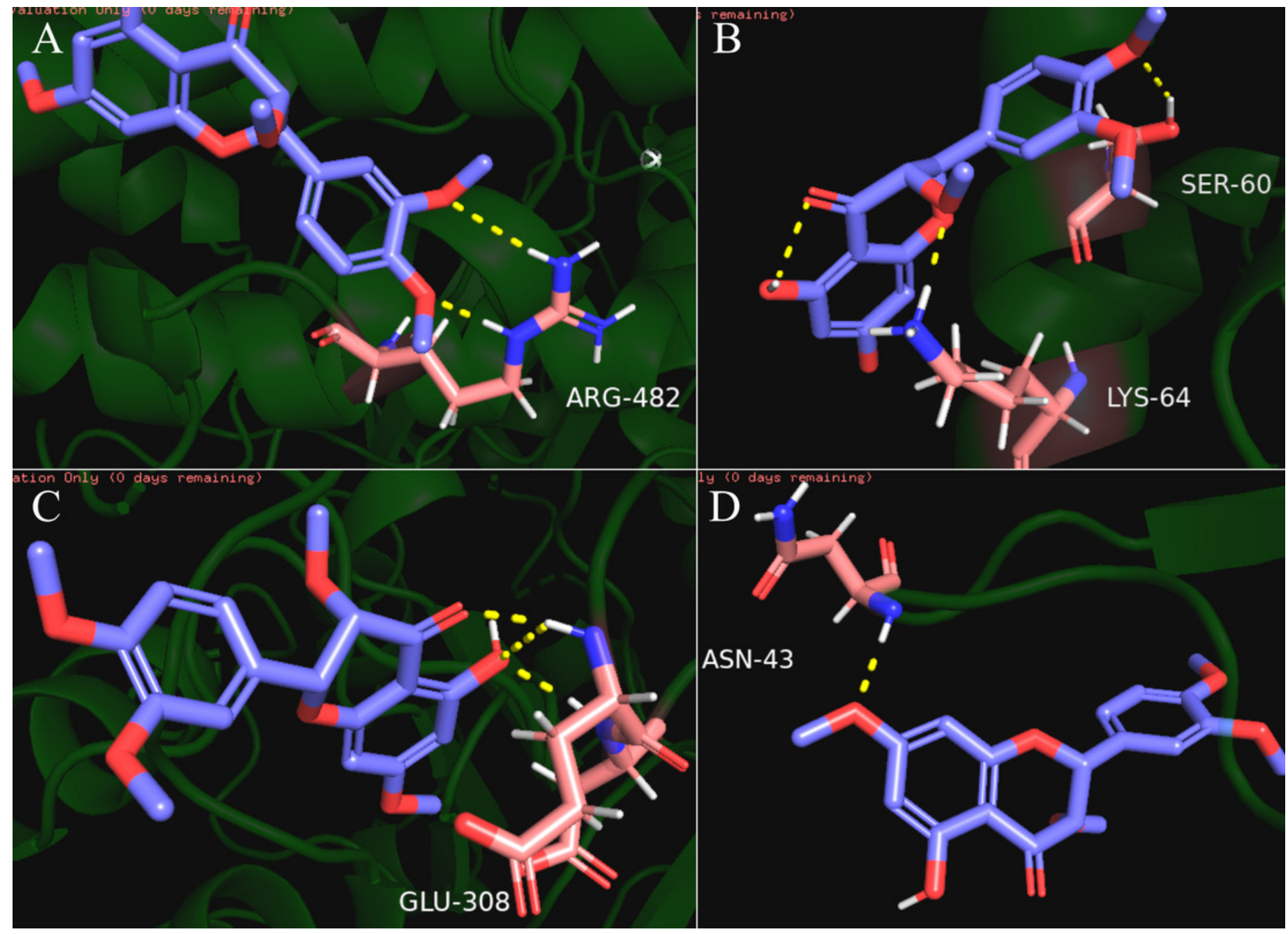




\section{Table $\mathbf{1}$ (on next page)}

Active ingredients and parameters of SiNiSan. 
ID

MOL000358

MOL000492

MOL001919

MOL001924

MOL000449

MOL000490

MOL001645

MOL004598

MOL004609

MOL004653

MOL004718

MOL013187

MOL000239

MOL000392

MOL000417

MOL000497

MOL000500

MOL001484

MOL001792

MOL002311
Herb

Baishao

Baishao

$(+)$-catechin

(3S,5R,8R,9R,10S,14S)-3,17-dihydroxy-4,4,8,10,14-

Baishao

pentamethyl-2,3,5,6,7,9-hexahydro-1H-

cyclopenta[a]phenanthrene-15,16-dione

Baishao

paeoniflorin

Stigmasterol

petunidin

Linoleyl acetate

Areapillin

$(+)$-Anomalin

$\alpha$-spinasterol

Cubebin

Jaranol

formononetin

Calycosin

licochalconea

Vestitol

Inermine

DFV

Glycyrol
43.56

0.53

53.87

43.83

30.05

42.1

31.97

48.96

DL

0.75

0.24

$\begin{array}{cc}\mathrm{OB}(\%) & \mathrm{DL} \\ 36.91 & 0.75 \\ & \\ 54.83 & 0.24\end{array}$

0.76

0.31

0.2

0.59

0.41

$46.06 \quad 0.66$

$42.98 \quad 0.76$

$57.13 \quad 0.64$

$50.83 \quad 0.29$

$69.67 \quad 0.21$

$\begin{array}{ll}47.75 & 0.24\end{array}$

$40.79 \quad 0.29$

$\begin{array}{ll}74.66 & 0.21\end{array}$

$32.76 \quad 0.18$

$90.78 \quad 0.67$ $\begin{array}{ll}75.18 & 0.54\end{array}$ 


\begin{tabular}{|c|c|c|c|c|}
\hline MOL002565 & Gancao & Medicarpin & 49.22 & 0.34 \\
\hline MOL003656 & Gancao & Lupiwighteone & 51.64 & 0.37 \\
\hline MOL003896 & Gancao & 7-Methoxy-2-methyl isoflavone & 42.56 & 0.2 \\
\hline MOL004805 & Gancao & $\begin{array}{l}\text { (2S)-2-[4-hydroxy-3-(3-methylbut-2-enyl)phenyl]-8,8- } \\
\text { dimethyl-2,3-dihydropyrano[2,3-f]chromen-4-one }\end{array}$ & 31.79 & 0.72 \\
\hline MOL004806 & Gancao & euchrenone & 30.29 & 0.57 \\
\hline MOL004808 & Gancao & glyasperin B & 65.22 & 0.44 \\
\hline MOL004810 & Gancao & glyasperin F & 75.84 & 0.54 \\
\hline MOL004811 & Gancao & Glyasperin C & 45.56 & 0.4 \\
\hline MOL004814 & Gancao & Isotrifoliol & 31.94 & 0.42 \\
\hline MOL004815 & Gancao & $\begin{array}{l}\text { (E)-1-(2,4-dihydroxyphenyl)-3-(2,2-dimethylchromen-6- } \\
\text { yl)prop-2-en-1-one }\end{array}$ & 39.62 & 0.35 \\
\hline MOL004820 & Gancao & kanzonols W & 50.48 & 0.52 \\
\hline MOL004824 & Gancao & $\begin{array}{l}\text { (2S)-6-(2,4-dihydroxyphenyl)-2-(2-hydroxypropan-2-yl)-4- } \\
\text { methoxy-2,3-dihydrofuro[3,2-g]chromen-7-one }\end{array}$ & 60.25 & 0.63 \\
\hline MOL004827 & Gancao & Semilicoisoflavone B & 48.78 & 0.55 \\
\hline MOL004828 & Gancao & Glepidotin A & 44.72 & 0.35 \\
\hline MOL004829 & Gancao & Glepidotin B & 64.46 & 0.34 \\
\hline ID & Herb & Compound & $\mathrm{OB}(\%)$ & $\mathrm{DL}$ \\
\hline MOL004833 & Gancao & Phaseolinisoflavan & 32.01 & 0.45 \\
\hline MOL004835 & Gancao & Glypallichalcone & 61.6 & 0.19 \\
\hline MOL004838 & Gancao & 8-(6-hydroxy-2-benzofuranyl)-2,2-dimethyl-5-chromenol & 58.44 & 0.38 \\
\hline MOL004841 & Gancao & Licochalcone B & 76.76 & 0.19 \\
\hline MOL004848 & Gancao & licochalcone $\mathrm{G}$ & 49.25 & 0.32 \\
\hline
\end{tabular}


MOL004849

MOL004855

MOL004856

MOL004857

MOL004863

MOL004864

MOL004866

MOL004879

MOL004882

MOL004883

MOL004884

MOL004885

MOL004891

MOL004898

MOL004903

MOL004904

MOL004907

MOL004908

MOL004910
Gancao

Gancao

Gancao

Gancao

Gancao

Gancao

Gancao

Gancao

Gancao

Gancao

Gancao

Gancao

Gancao

Gancao

Gancao

Gancao

Gancao

Gancao

Gancao 3-(2,4-dihydroxyphenyl)-8-(1,1-dimethylprop-2-enyl)-7hydroxy-5-methoxy-coumarin

59.62

0.43

63.58

0.47

Licoricone

Gancaonin A

51.08

0.4

48.79

0.45

3-(3,4-dihydroxyphenyl)-5,7-dihydroxy-8-(3-methylbut-2enyl)chromone

66.37

0.41

5,7-dihydroxy-3-(4-methoxyphenyl)-8-(3-methylbut-2enyl)chromone

30.49

0.41

2-(3,4-dihydroxyphenyl)-5,7-dihydroxy-6-(3-methylbut-2enyl)chromone

44.15

Glycyrin

52.61

0.47

Licocoumarone

33.21

0.36

Licoisoflavone

41.61

0.42

Licoisoflavone B

38.93

0.55

licoisoflavanone

52.47

0.54

shinpterocarpin

80.3

0.73

(E)-3-[3,4-dihydroxy-5-(3-methylbut-2-enyl)phenyl]-1-(2,4dihydroxyphenyl)prop-2-en-1-one

65.69

0.74

liquiritin

licopyranocoumarin

80.36

0.65

Glyzaglabrin

61.07

0.35

Glabridin

53.25

0.47

Glabranin

52.9

0.31 


\begin{tabular}{|c|c|c|c|c|}
\hline MOL004911 & Gancao & Glabrene & 46.27 & 0.44 \\
\hline MOL004912 & Gancao & Glabrone & 52.51 & 0.5 \\
\hline MOL004913 & Gancao & 1,3-dihydroxy-9-methoxy-6-benzofurano[3,2-c]chromenone & 48.14 & 0.43 \\
\hline MOL004914 & Gancao & $\begin{array}{l}\text { 1,3-dihydroxy-8,9-dimethoxy-6-benzofurano[3,2- } \\
\text { c]chromenone }\end{array}$ & 62.9 & 0.53 \\
\hline MOL004915 & Gancao & Eurycarpin A & 43.28 & 0.37 \\
\hline MOL004924 & Gancao & (-)-Medicocarpin & 40.99 & 0.95 \\
\hline MOL004935 & Gancao & Sigmoidin-B & 34.88 & 0.41 \\
\hline MOL004941 & Gancao & (2R)-7-hydroxy-2-(4-hydroxyphenyl)chroman-4-one & 71.12 & 0.18 \\
\hline MOL004945 & Gancao & $\begin{array}{l}\text { (2S)-7-hydroxy-2-(4-hydroxyphenyl)-8-(3-methylbut-2- } \\
\text { enyl)chroman-4-one }\end{array}$ & 36.57 & 0.32 \\
\hline MOL004948 & Gancao & Isoglycyrol & 44.7 & 0.84 \\
\hline ID & Herb & Compound & $\mathrm{OB}(\%)$ & $\mathrm{DL}$ \\
\hline MOL004949 & Gancao & Isolicoflavonol & 45.17 & 0.42 \\
\hline MOL004957 & Gancao & $\mathrm{HMO}$ & 38.37 & 0.21 \\
\hline MOL004959 & Gancao & 1-Methoxyphaseollidin & 69.98 & 0.64 \\
\hline MOL004961 & Gancao & Quercetin der. & 46.45 & 0.33 \\
\hline MOL004966 & Gancao & 3'-Hydroxy-4'-O-Methylglabridin & 43.71 & 0.57 \\
\hline MOL004974 & Gancao & 3'-Methoxyglabridin & 46.16 & 0.57 \\
\hline MOL004978 & Gancao & $\begin{array}{l}\text { 2-[(3R)-8,8-dimethyl-3,4-dihydro-2H-pyrano[6,5- } \\
\text { f]chromen-3-yl]-5-methoxyphenol }\end{array}$ & 36.21 & 0.52 \\
\hline MOL004980 & Gancao & Inflacoumarin A & 39.71 & 0.33 \\
\hline MOL004988 & Gancao & Kanzonol F & 32.47 & 0.89 \\
\hline MOL004989 & Gancao & 6-prenylated eriodictyol & 39.22 & 0.41 \\
\hline
\end{tabular}




\begin{tabular}{|c|c|c|c|c|}
\hline MOL004990 & Gancao & 7,2',4'-trihydroxy -5 -methoxy-3-arylcoumarin & 83.71 & 0.27 \\
\hline MOL004991 & Gancao & 7-Acetoxy-2-methylisoflavone & 38.92 & 0.26 \\
\hline MOL004993 & Gancao & 8-prenylated eriodictyol & 53.79 & 0.4 \\
\hline MOL005000 & Gancao & Gancaonin G & 60.44 & 0.39 \\
\hline MOL005001 & Gancao & Gancaonin $\mathrm{H}$ & 50.1 & 0.78 \\
\hline MOL005003 & Gancao & Licoagrocarpin & 58.81 & 0.58 \\
\hline MOL005007 & Gancao & Glyasperins M & 72.67 & 0.59 \\
\hline MOL005008 & Gancao & Glycyrrhiza flavonol A & 41.28 & 0.6 \\
\hline MOL005012 & Gancao & Licoagroisoflavone & 57.28 & 0.49 \\
\hline MOL005016 & Gancao & Odoratin & 49.95 & 0.3 \\
\hline MOL005017 & Gancao & Phaseol & 78.77 & 0.58 \\
\hline MOL005018 & Gancao & Xambioona & 54.85 & 0.87 \\
\hline MOL005020 & Gancao & dehydroglyasperins $\mathrm{C}$ & 53.82 & 0.37 \\
\hline MOL000006 & Zhishi & luteolin & 36.16 & 0.25 \\
\hline MOL001798 & Zhishi & neohesperidin_qt & 71.17 & 0.27 \\
\hline MOL001803 & Zhishi & Sinensetin & 50.56 & 0.45 \\
\hline MOL001941 & Zhishi & Ammidin & 34.55 & 0.22 \\
\hline MOL002914 & Zhishi & Eriodyctiol (flavanone) & 41.35 & 0.24 \\
\hline MOL005100 & Zhishi & $\begin{array}{c}\text { 5,7-dihydroxy-2-(3-hydroxy-4-methoxyphenyl)chroman-4- } \\
\text { one }\end{array}$ & 47.74 & 0.27 \\
\hline MOL005828 & Zhishi & nobiletin & 61.67 & 0.52 \\
\hline MOL005849 & Zhishi & didymin & 38.55 & 0.24 \\
\hline MOL007879 & Zhishi & Tetramethoxyluteolin & 43.68 & 0.37 \\
\hline
\end{tabular}




\begin{tabular}{|c|c|c|c|c|}
\hline MOL009053 & Zhishi & $\begin{array}{l}\text { 4-[(2S,3R)-5-[(E)-3-hydroxyprop-1-enyl]-7-methoxy-3- } \\
\text { methylol-2,3-dihydrobenzofuran-2-yl]-2-methoxy-phenol }\end{array}$ & 50.76 & 0.39 \\
\hline MOL013277 & Zhishi & Isosinensetin & 51.15 & 0.44 \\
\hline MOL013279 & Zhishi & 5,7,4'-Trimethylapigenin & 39.83 & 0.3 \\
\hline MOL013430 & Zhishi & Prangenin & 43.6 & 0.29 \\
\hline MOL013435 & Zhishi & poncimarin & 63.62 & 0.35 \\
\hline MOL013436 & Zhishi & isoponcimarin & 63.28 & 0.31 \\
\hline MOL013437 & Zhishi & 6-Methoxy aurapten & 31.24 & 0.3 \\
\hline ID & Herb & Compound & $\mathrm{OB}(\%)$ & DL \\
\hline MOL004828 & Gancao & Glepidotin A & 44.72 & 0.35 \\
\hline MOL004829 & Gancao & Glepidotin B & 64.46 & 0.34 \\
\hline MOL004833 & Gancao & Phaseolinisoflavan & 32.01 & 0.45 \\
\hline MOL004835 & Gancao & Glypallichalcone & 61.6 & 0.19 \\
\hline MOL004838 & Gancao & 8-(6-hydroxy-2-benzofuranyl)-2,2-dimethyl-5-chromenol & 58.44 & 0.38 \\
\hline MOL004841 & Gancao & Licochalcone B & 76.76 & 0.19 \\
\hline MOL004848 & Gancao & licochalcone $\mathrm{G}$ & 49.25 & 0.32 \\
\hline MOL004849 & Gancao & $\begin{array}{c}\text { 3-(2,4-dihydroxyphenyl)-8-(1,1-dimethylprop-2-enyl)-7- } \\
\text { hydroxy-5-methoxy-coumarin }\end{array}$ & 59.62 & 0.43 \\
\hline MOL004855 & Gancao & Licoricone & 63.58 & 0.47 \\
\hline MOL004856 & Gancao & Gancaonin A & 51.08 & 0.4 \\
\hline MOL000098 & Multi-herb & quercetin & 46.43 & 0.28 \\
\hline MOL000354 & Multi-herb & isorhamnetin & 49.6 & 0.31 \\
\hline MOL000359 & Multi-herb & sitosterol & 36.91 & 0.75 \\
\hline
\end{tabular}


MOL000422

MOL004328
Multi-herb

Multi-herb kaempferol

naringenin
41.88

59.29
0.24

0.21 


\section{Table 2 (on next page)}

The results of KEGG pathway enrichment analysis of 64 intersection HCC genes. 


\begin{tabular}{|c|c|c|c|}
\hline ID & Description & pvalue & geneID \\
\hline hsa04110 & Cell cycle & 4.70E-06 & $\begin{array}{c}\text { CDK1/CCNA2/CHEK1/MYC/CCN } \\
\text { B1/CHEK2/E2F1/E2F2/PCNA }\end{array}$ \\
\hline hsa04115 & p53 signaling pathway & $9.62 \mathrm{E}-06$ & $\begin{array}{c}\text { BAX/CDK1/CHEK1/CCNB1/SERPI } \\
\text { NE1/CHEK2/IGFBP3 }\end{array}$ \\
\hline hsa00982 & $\begin{array}{l}\text { Drug metabolism - cytochrome } \\
\qquad \text { P450 }\end{array}$ & $9.82 \mathrm{E}-05$ & $\begin{array}{c}\text { CYP3A4/CYP1A2/GSTM1/ADH1C/ } \\
\text { MAOB/MAOA }\end{array}$ \\
\hline hsa00140 & Steroid hormone biosynthesis & 0.000424164 & $\begin{array}{c}\text { CYP3A4/CYP1A2/AKR1C3/SULT1 } \\
\text { E1/CYP19A1 }\end{array}$ \\
\hline hsa00350 & Tyrosine metabolism & 0.000521784 & ADH1C/MAOB/MAOA/GOT1 \\
\hline hsa01522 & Endocrine resistance & 0.000536494 & BAX/JUN/ESR1/FOS/E2F1/E2F2 \\
\hline hsa00360 & Phenylalanine metabolism & 0.000695885 & MAOB/MAOA/GOT1 \\
\hline hsa00380 & Tryptophan metabolism & 0.000945976 & CYP1A2/CAT/MAOB/MAOA \\
\hline hsa04020 & Calcium signaling pathway & 0.000947819 & $\begin{array}{l}\text { ADRA1A/CHRM2/ADRA1B/ADRB } \\
\text { 2/PRKCA/NOS2/PPP3CA/PRKCB }\end{array}$ \\
\hline hsa00330 & Arginine and proline metabolism & 0.00182834 & NOS2/MAOB/MAOA/GOT1 \\
\hline hsa04064 & NF-kappa B signaling pathway & 0.004651344 & $\begin{array}{c}\text { CD14/PTGS2/PRKCB/PARP1/CXC } \\
\text { L2 }\end{array}$ \\
\hline hsa04066 & HIF-1 signaling pathway & 0.005678296 & $\begin{array}{c}\mathrm{PRKCA} / \mathrm{NOS} 2 / \mathrm{HMOX} 1 / \mathrm{PRKCB} / \mathrm{SE} \\
\text { RPINE1 }\end{array}$ \\
\hline hsa01524 & Platinum drug resistance & 0.007222453 & BAX/GSTM1/BIRC5/TOP2A \\
\hline hsa00980 & $\begin{array}{l}\text { Metabolism of xenobiotics by } \\
\text { cytochrome P450 }\end{array}$ & 0.008704905 & CYP3A4/CYP1A2/GSTM1/ADH1C \\
\hline
\end{tabular}




\section{Table 3(on next page)}

The results of molecular docking between quercetin and AR, BAX, CHEK2, ESR1, NOS2, PTGS2.

Including binding energy, the inhibition constant, the total internal energy $(V d w-h b-d e s o l v+$ electrostatic energy) between the receptor and the ligand. 


\begin{tabular}{|c|c|c|c|c|c|c|}
\hline $\begin{array}{l}\text { Component } \\
\text { ligand }\end{array}$ & Target & PDB ID & $\begin{array}{c}\text { Hbonding atoms of } \\
\text { target }\end{array}$ & $\begin{array}{l}\text { Hbinding } \\
\text { energy } \\
(\mathrm{Kcal} / \mathrm{mol})\end{array}$ & $\begin{array}{l}\text { Inhib } \\
\text { constant }\end{array}$ & $\begin{array}{c}\text { Vdw-hb- } \\
\text { desolv }+ \\
\text { electrostatic } \\
\text { energy } \\
(\mathrm{Kcal} / \mathrm{mol})\end{array}$ \\
\hline Quercetin & AR & 4WEV & $\begin{array}{l}\text { GLU308:HN; } \\
\text { ASP309:HN }\end{array}$ & -4.24 & $775.54 \mathrm{uM}$ & -6.04 \\
\hline Quercetin & PTGS2 & $5 \mathrm{KIR}$ & ASN43:H & -4.11 & $976.06 \mathrm{uM}$ & -5.9 \\
\hline Quercetin & PTGS2 & $5 \mathrm{KIR}$ & GLY66:H & -3.93 & $1.32 \mathrm{mM}$ & -5.72 \\
\hline Quercetin & AR & 2Q7K & $\begin{array}{c}\text { ARG788:HH11; } \\
\text { HIS789:HD1; } \\
\text { GLN792:HE22 }\end{array}$ & -3.36 & $3.45 \mathrm{mM}$ & -5.14 \\
\hline Quercetin & CHEK2 & $2 \mathrm{CN} 8$ & ARG474:HH22 & -3.36 & $3.44 \mathrm{mM}$ & -5.15 \\
\hline Quercetin & PTGS2 & $5 \mathrm{KIR}$ & $\begin{array}{c}\text { ARG77:HE; } \\
\text { ARG77:HH11 }\end{array}$ & -3.2 & $6.28 \mathrm{mM}$ & -4.79 \\
\hline Quercetin & PTGS2 & $5 \mathrm{KIR}$ & ARG44:HE; CYS47:H & -3.19 & $4.55 \mathrm{mM}$ & -4.99 \\
\hline Quercetin & NOS2 & $5 \mathrm{XN} 3$ & GLU219:HN & -3.17 & $4.78 \mathrm{mM}$ & -4.96 \\
\hline Quercetin & PTGS2 & $5 \mathrm{KIR}$ & LYS83:HZ1 & -3.11 & $3.72 \mathrm{mM}$ & -5.1 \\
\hline Quercetin & AR & 2Q7K & $\begin{array}{c}\text { ARG788:HH11; } \\
\text { ARG788:HH21; } \\
\text { HIS789:HD1; } \\
\text { GLN792:HE22 }\end{array}$ & -3.05 & $5.8 \mathrm{mM}$ & -4.85 \\
\hline Quercetin & CHEK2 & 4A9U & ARG482:HE & -3.05 & $5.78 \mathrm{mM}$ & -4.84 \\
\hline Quercetin & CHEK2 & $2 \mathrm{CN} 8$ & LYS444:HZ1 & -3.04 & $5.86 \mathrm{mM}$ & -4.84 \\
\hline Quercetin & BAX & 4BD7 & $\begin{array}{l}\text { SER60:HG; } \\
\text { LYS64:HZ3 }\end{array}$ & -3.00 & $3.83 \mathrm{mM}$ & -5.09 \\
\hline Quercetin & PTGS2 & $5 \mathrm{KIR}$ & HIS90:HD1 & -2.91 & $6.7 \mathrm{mM}$ & -4.75 \\
\hline
\end{tabular}




\begin{tabular}{ccccccc}
\hline Quercetin & CHEK2 & 2CN8 & LYS464:HZ2 & -2.78 & $9.17 \mathrm{mM}$ & -4.57 \\
Quercetin & AR & 5BXJ & ARG39:HH12; & -2.23 & $23.17 \mathrm{mM}$ & -4.02 \\
Quercetin & AR & 5BXJ & ARG39:HH22; & -2.19 & $24.99 \mathrm{mM}$ & -3.98 \\
Quercetin & NOS2 & 4NOS & LYS123:HZ3 & -1.68 & $58.96 \mathrm{mM}$ & -3.47 \\
Quercetin & BAX & 4BD6 & LYS119:HZ1 & -1.67 & $59.9 \mathrm{mM}$ & -3.46 \\
Quercetin & ESR1 & 6KN5 & LYS996:HZ1 & -1.64 & $63.15 \mathrm{mM}$ & -3.43 \\
Quercetin & BAX & 4BD6 & SER72: HG & -1.59 & $68.29 \mathrm{mM}$ & -3.38 \\
Quercetin & ESR1 & $4 X I 3$ & LYS467:HZ1 & -1.59 & $68.65 \mathrm{mM}$ & -3.53 \\
\hline
\end{tabular}

1 